\title{
CSA Discounting: Impacts on Pricing and Risk of Commodity Derivatives
}

\author{
R. Abbate \\ Financial Engineering \& Quantitative Analysis, SunGard, New York, United States
}

Received 16 May 2014; revised 10 June 2014; accepted 28 June 2014

Copyright (C) 2014 by author and Scientific Research Publishing Inc.

This work is licensed under the Creative Commons Attribution International License (CC BY). http://creativecommons.org/licenses/by/4.0/

(c) (i) 0pen Access

\section{Abstract}

The global financial crisis of 2007-2008 caused market practitioners to reassess the way in which financial derivative contracts had been priced during the preceding thirty years. The purpose of this paper is to examine the evolving practice of pricing and hedging commodity derivative contracts according to the terms of the Credit Support Annex (CSA). Using a series of case studies, we price crude oil swaps and Asian options in the pre-crisis, peak-crisis, post-crisis and recent market environments under two different frameworks: LIBOR discounting and CSA discounting (also referred to in a less general form as "OIS discounting", which incorporates nearly risk-free interest rates). We also compute the widely used first-order and second-order Greek sensitivities. In each market environment, we shift the forward prices and implied volatilities crude oil and re-compute the trades' valuation and Greek sensitivities at each incremental increase or decrease in price or implied volatility. Under each discounting framework, we quantify the change in trade valuation and Greek sensitivities that results from switching from LIBOR discounting to CSA discounting. The impact on the valuation and Greek sensitivities of a swap and an Asian option as the result of adopting CSA discounting can be significant under certain market conditions. There is likely to be larger impact on directional portfolios containing transactions that hedge either consumption or production (e.g. end users). Ceteris paribus, the impact on portfolio valuation and risk is likely to be limited for market participants (e.g. banks) with hedged portfolios that contain a large number of offsetting positions. Even though we focus our analysis on crude oil derivative contracts, the results easily extend to other asset classes such as natural gas, refined products, agriculture, metals, etc.

\section{Keywords}

LIBOR, OIS, CSA, Discounting, Commodities, Crude Oil, Derivatives, Valuation, Risk 


\section{Introduction}

The financial crisis, which began in August 2007, triggered a paradigm shift in the way many market participants approach one of the most fundamental aspects of derivatives pricing and risk management: cash flow discounting. In recent years, the classical approach to discounting has been abandoned by many participants in favor of a technique that incorporates the collateralization required by Credit Support Annex (CSA) agreements. Many practitioners now question the appropriateness of using LIBOR as the proxy for the risk-neutral discount rate.

Regulations such as the Dodd-Frank Wall Street Reform and Consumer Protection Act of 2010, the Third Basel Accord and the Solvency II Directive, along with accounting rules such as Accounting Standards Codification Topic 820, have mandated more accurate counterparty risk valuation. In the aftermath of the financial crisis, it is necessary to value the credit component of each transaction in order to accurately value derivatives contracts.

\section{Literature Review}

Earlier works examining the pricing of counterparty credit risk invarious asset classes include, for example, (Sorenson, 1994), who show that the counterparty credit risk on a swap can be expressed as a function of swaptions with various exercise dates and (Brigo, 2005), who calculate the Credit Valuation Adjustment (CVA) assuming unilateral counterparty credit risk. Earlier works treating the commodity asset classes include, for example, (Canabarro, 2005), who analyze the topic from a capital adequacy and risk management perspective. There are, to our knowledge, no published works that specifically quantify the impacts to valuation and risk from switching from LIBOR $^{1}$ to OIS discounting across commonly traded instruments in the commodity derivatives markets.

\section{A Brief Refresher on Risk-Neutral Pricing}

Risk-neutral pricing is a technique widely used in mathematical finance to price contingent claims. In this section, we provide the reader with a brief review in order to motivate discussion on arbitrage-free pricing in Section 9 of this paper.

A market is considered to be complete if any contingent claim can be replicated by an admissible, self-financing trading strategy referred to as a replicating portfolio. The replicating portfolio is constructed from primary securities such as stocks and bonds, the market prices of which are unique. The price of the replicating portfolio is identical to all agents in the market, and is therefore independent of any assumptions of risk preferences, either averse or seeking. Regardless of their risk preferences, market participants will eliminate any discrepancy between the price of the replicating portfolio and the prices of its underlying primary securities by engaging in arbitrage trades.

Typically, the probabilities of events occurring are expressed in terms of the physical (i.e. "realworld") probability measure, which we denote as $\mathbb{P}$ in the probability space $(\Omega, \mathcal{F}, \mathbb{P})$. However, a problem arises because market participants discount the risk of a contract with varying interest rates according to their individual risk preferences. Therefore, we apply Girsanov's theorem and use a probability measure, which we denote as $\mathbb{Q}$, and is equivalent to $\mathbb{P}$, under which market participants are insensitive to risk (i.e. risk-neutral).This is a powerful and tractable technique because it allows us to discount cash flows at the risk-free rate $r$ when computing expectations under the probability measure $\mathbb{Q}$.

A martingale is the mathematical formalization of a fair game (e.g. coin flip). The process $X$ is said to be a martingale if

$$
E\left[X_{t+i} \mid \mathcal{F}_{t}\right]=X_{t} \text {, for all } i \text { and } t \geq 0
$$

If we model the prices of financial contracts as martingales, then we can imply market participants are unable

${ }^{1}$ LIBOR, an acronym for London Inter-Bank Offered Rate, is the interest rate at which an individual contributor panel bank is able to borrow unsecured funds, were it to do so by asking for and then accepting interbank offers in reasonable market size, just prior to 11:00 a.m. London time. On behalf of the ICE Benchmark Administration (previously the British Bankers' Association), Thomson Reuters polls a minimum of 8 and a maximum of 16 large global banks for their interest rates across a range of seven maturities in five major currencies. The maturities range from overnight to 12 months, with the 1-month, 3-month and 6-month tenors widely considered the benchmarks to which many interest rate derivative contracts are indexed. The top $25 \%$ and bottom $25 \%$ of the contributor banks' submissions are discarded and the middle $50 \%$ of submissions is then averaged. This truncated mean becomes the LIBOR fixing for a given maturity. LIBOR represents the cost of funds for large global banks with a credit rating resembling "AA". 
to consistently profit by trading these financial contracts.

Under the risk-neutral probability measure $\mathbb{Q}$, discounted prices are martingales

$$
P(0, t) X_{t}=E^{\mathbb{Q}}\left[P(0, T) X_{T} \mid \mathcal{F}_{t}\right]
$$

Rearranging Equation (3.2) and using discount factor $P(0, t)=\frac{1}{(1+r)^{t}}$, we have

$$
(1+r)^{T-t}=E^{\mathbb{Q}}\left[\frac{X_{T}}{X_{t}} \mid \mathcal{F}_{t}\right]
$$

With the drift removed, the expected value of the return on $X$ from time $t$ to maturity $T$ is equal to the risk-free rate $r$.

There is no arbitrage if and only if a risk-neutral (i.e. equivalent martingale) measure $Q$ exists. This is the First Fundamental Theorem of Asset Pricing. The market is complete if and only if exactly one risk-neutral martingale measure, $Q$, exists. This is the Second Fundamental Theorem of Asset Pricing. Stated differently, if the market is arbitrage-free and complete, then the time $t$ value of a contingent claim $X$ is equal to the time $t$ value of a portfolio that replicates $X$. The arbitrage-free price of any financial contract in a complete market is equal to the expected value of the future payoff under the risk-neutral measure, discounted at the risk-free rate.

If we denote $V_{t}$ as the replicating portfolio, then the arbitrage-free price of any contingent claim $C$ is given by

$$
V_{t}=\mathrm{e}^{-r(T-t)} \mathbb{E}^{Q}\left[C \mid \mathcal{F}_{t}\right], 0 \leq t \leq T
$$

where $\mathbb{E}^{Q}$ denotes the conditional expectation under the risk-neutral measure $\mathbb{Q}, r$ denotes the risk-free interest rate and $\mathcal{F}_{t}$ denotes the information filtration at any time $t$.

A position in the derivative contract can be hedged with a position in $\Delta$ units of the underlying (Black \& Scholes, 1973). In theory, this results in a riskless portfolio. However, the theory relies on a number of assumptions. One key assumption is that market participants are able to continuously and completely hedge away all of the risk. In practice, this is not possible because, inter alia, markets are incomplete. Therefore, according to the Second Fundamental Theorem of Asset Pricing, the preference for risk must be reintroduced into derivatives valuations, resulting in individual assessments of risk. The Law of One Price, an economic concept which states that two assets with identical cash flows and risk characteristics should have the same price in a market that is complete and arbitrage-free, no longer holds. Another assumption is the ability of market participants to borrow limitless amounts at the risk-free rate. This, too, is not possible in practice.

The reader is referred to Appendix A for a derivation of the pricing equation for a financial derivative contract.

\section{The Classical Approach to Pricing Rendered Obsolete}

As the interest rate swap market developed in the 1980s, LIBOR was perceived as the benchmark that most appropriately reflected the funding cost of interbank transactions, and was therefore used as the proxy for the "risk-free" rate. Collateralization had not yet been widely adopted. Best practices prescribed the use of a LIBOR zero-coupon curve to discount the cash flows of these non-collateralized derivatives contracts.

Market participants typically calibrated a single interest rate curve to liquid market products such as cash deposits, forward rate agreements, short-term interest rate futures (e.g. Eurodollar futures) and/or interest rate swaps. An interpolation technique was chosen to solve for interior points. Convexity adjustments were applied to the interest rates implied by the short-term futures contracts. The curve was adjusted for turn-of-year effects. The discount factors used in derivatives pricing were then derived from this zero-coupon curve. Expected cash flows were discounted from this single curve based on no-arbitrage assumptions. There was little variation between implementations and the pricing results were consistent among market practitioners. Importantly, the prices of derivative contracts in developed markets quoted to clients by different banks were generally consistent.

Prior to the financial crisis, LIBOR was considered to be the risk-free rate, as well as the rate at which banks could fund themselves. There was little distinction between the risk-free rate used for the purposes of discounting and a bank's cost of funding. The effects of credit risk, liquidity risk, collateral agreements and funding costs 
were largely ignored. The primary focus was to price the market risk of transactions. Collateralized and noncollateralized derivatives transactions had traditionally been priced using the LIBOR zero-coupon curve.

The financial crisis has taught us that no institution is "too big to fail" and that LIBOR is a poor proxy for the risk-neutral discount rate. Market participants have become keenly aware of the importance of credit and liquidity risks. The key assumption that firms fund and invest at LIBOR (known as the "self-discounting" framework) has been rendered obsolete. The modern approach to yield curve modeling requires one curve to project forward rates and a separate curve to discount cash flows. The forward rates are conditional on the rates used for discounting. By comparison, the forward curves used to price commodity derivatives are not conditional on the rates used for discounting. Therefore, in this paper, we concern ourselves only with the appropriate curve for cash flow discounting.

Meanwhile, the regulatory landscape continues to evolve. The Dodd-Frank Act and European Market Infrastructure Regulation (EMIR) require all eligible swaps to be centrally cleared. For swaps that are not centrally cleared, the Dodd-Frank Act mandates the use of collateralization. Under Basel III, which will be phased in between the years 2014 and 2018, risk weights on collateralized transactions differ significantly from risk weights on uncollateralized transactions. Collateralized transactions receive essentially a zero risk weighting, while noncollateralized transactions receive a risk weighting commensurate with the risk weight of the counterparty.

\section{Federal Funds}

The interest rate at which depository institutions lend excess reserve balances to each other on an overnight basis is referred to as the Federal Funds rate. Banks raise overnight unsecured financing in U.S. dollars in the form of Federal Funds. The majority of Federal Funds loans are transacted through inter-dealer brokers. Many such brokers report the principal amount loaned at each interest rate to the Federal Reserve Bank of New York each day. Using the brokers' submissions, the Federal Reserve Bank of New York calculates a weighted average interest rate paid on the Federal Funds loans each day. This weighted average interest rate is referred to as the "effective Federal Funds rate" and is published daily on a $\mathrm{T}+1$ basis at approximately 8:00 a.m. EST. The Federal Reserve Bank keeps the effective Federal Funds rate close to the so-called "target" interest rate by buying or selling securities in open market operations.

\section{Overnight Index Swaps}

An Overnight Index Swap (OIS) is a fixed-for-floating interest rate swap whose floating leg references an overnight index that resets each day during the specified term of the swap. Interest on the floating leg is computed through daily compounding (i.e. geometric averaging) of the overnight index. The floating rate index is typically the overnight secured lending rate between banks. Examples include the effective Federal Funds rate in U.S. dollars or its equivalent in other currencies ${ }^{2}$.

The OIS is a measure of market participants' expectations of the effective Federal Funds rate during the term of the swap. The calculation of the payment on the floating leg is designed to replicate the aggregate amount of interest that would accrue from rolling over a sequence of overnight loans at the overnight rate.

For instance, let us assume that a bank borrows at a 3-month U.S. dollar LIBOR rate of 30 basis points. Let us further assume that this bank enters into a swap to receive 3-month U.S. dollar OIS at a rate of 10 basis points. The bank now has a borrowing cost equal to the effective Federal Funds rate plus 20 basis points. Although the OIS exposes the bank to fluctuations in the path of the effective Federal Funds rates, the bank can secure longerterm funding while still paying interest relatively near to the overnight rate. Market participants pay a premium for the term funding because the alternative would require the funds to be reinvested each day at a stochastic interest rate.

Overnight Index Swaps are available in most major currencies. In emerging currencies, however, the OIS market does not yet exist. In some currencies, the market for OIS exists but is illiquid. In these cases, it is possible to convert the U.S. dollar OIS curve using either foreign exchange forwards or swaps to build a synthetic foreign currency OIS curve. In cases of illiquidity (e.g. tenors greater than 5 years in most currencies), it is possible to use the basis swap between LIBOR and the underlying overnight rate to bootstrap the OIS curve to the desired tenor.

\footnotetext{
${ }^{2}$ Examples include the Euro Overnight Index Average (EONIA), the Sterling Overnight Index Average (SONIA), the Canadian Overnight Repo Rate (CORRA), the Uncollateralized Overnight Call Rate (MUTAN), the Tom-Next Index Swaps (TOIS), etc.
} 
LIBOR rates have been recognizable and observable benchmark interest rates for several decades. While the OIS market has existed for quite some time, the OIS rates tend to be less observable and less liquid than LIBOR rates. Additionally, the construction of the OIS curvet ends to be more nuanced than that of the LIBOR curve of like tenor. Although the daily tenor of OIS rates mitigates credit and liquidity risk, we wish to stress that OIS rates are not risk-free.

\section{Relationship between LIBOR and OIS}

The spread between LIBOR and OIS measures the health of the banking system. The spread is an indication of banks' perception of the credit condition of other financial institutions, along with the general availability of funds for lending purposes. The spread between LIBOR and OIS tends to widen when market participants become concerned about creditworthiness of financial institutions with a rating resembling AA (Figure 1).

Before the onset of the turmoil in the credit markets in 2007, the spread between 3-month U.S. dollar LIBOR and 3-month U.S. dollar OIS traded at approximately 7 basis points. In September 2007, the spread widened to 95 basis points after the Bank of England announced emergency funding to rescue the distressed Northern Rock. With the financial markets already unsettled by the collapse of Bear Stearns in March 2008, the bankruptcy of Lehman Brothers in September 2008 resulted in the spread widening to its peak of 364 basis points by October 2008. Banks had become unwilling to lend to each other amid widespread default concerns.

In the years following the financial crisis, the spread between 3-month U.S. dollar LIBOR and 3-month U.S. dollar OIS has tightened to more traditional levels but has not reverted to the negligible levels observed prior to the crisis. In Q1 2011, the spread rose from 15 basis points to a peak of 60 basis points on concerns over the default of certain European sovereign debt. In 2012, the median spread was 30 basis points. Since then, an elevated spread has persisted and reflects the revised view of market participants that LIBOR and OIS rates share different credit and liquidity characteristics.

The financial crisis impacted the structure and dynamics of the interest rate markets. Credit and liquidity concerns resulted in segmentation. Figure 2 depicts the spread between the spot 3-month U.S. dollar LIBOR fixing and the spot 6-month U.S. dollar LIBOR fixing. Rates that had been highly correlated diverged significantly.

The divergence between previously correlated rates was not limited to the short end of the term structure. Figure 3 depicts the behavior of the spread between the 2-year constant maturity fixed-for-floating LIBOR swap (CMS) and the 2-year constant maturity Treasury note (CMT), the so-called "TED spread".

Figure 4 depicts the behavior of the spread between 5-year CMS and 5-year CMT. Clearly, during times of financial markets stress, LIBOR is an inappropriate proxy for the "risk-free" rate.

Even prior to the financial crisis, many market participants had identified the OIS rates as the appropriate discount curve for collateralized transactions. However, for practical purposes, because the spread between 3month U.S. dollar LIBOR and 3-month U.S. dollar OIS was negligible and relatively stable prior to 2007, these market participants excluded this basis effect and used the LIBOR curve for pricing instead. As Figure 5

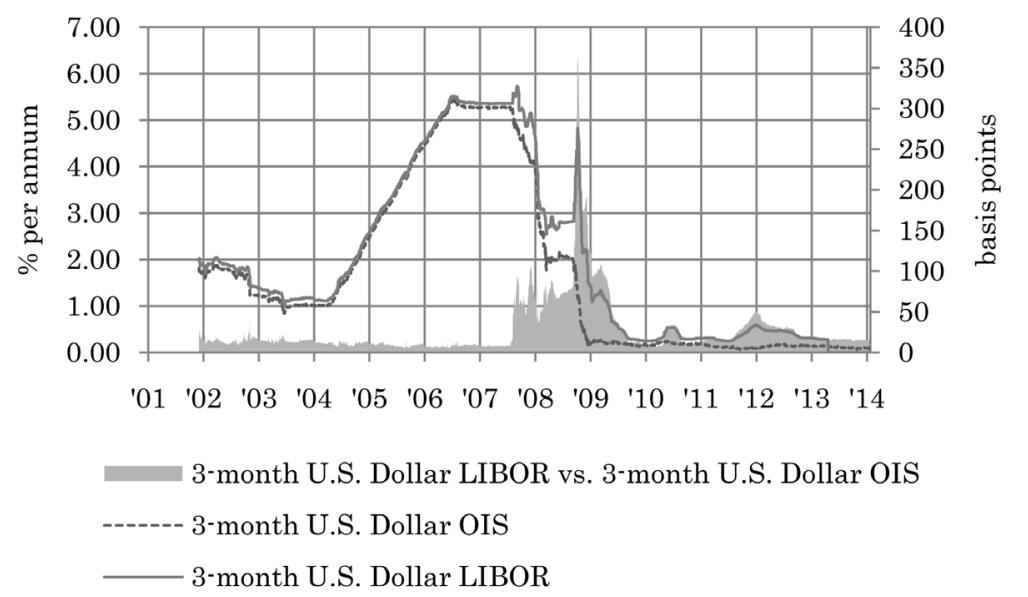

Figure 1. 3-month U.S. Dollar LIBOR vs. 3-month U.S. Dollar OIS. Source: Bloomberg, L.P. (2014). 


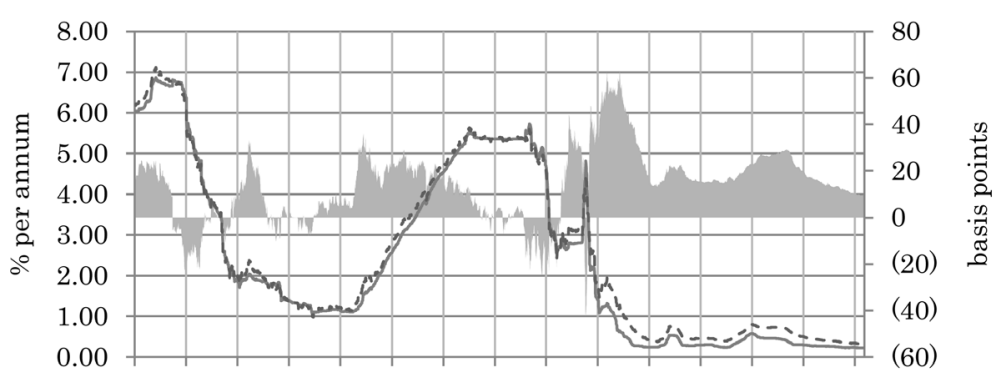

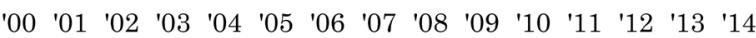

$$
\begin{aligned}
& \text { 3-month U.S. Dollar LIBOR vs. 6-month U.S. Dollar LIBOR } \\
& \text { - 3-month U.S. Dollar LIBOR } \\
& --- \text { 6-month U.S. Dollar LIBOR }
\end{aligned}
$$

Figure 2. 3-month U.S. Dollar LIBOR fixing vs. 6-month U.S. Dollar LIBOR fixing. Source: Federal Reserve Bank of St. Louis (FRED).

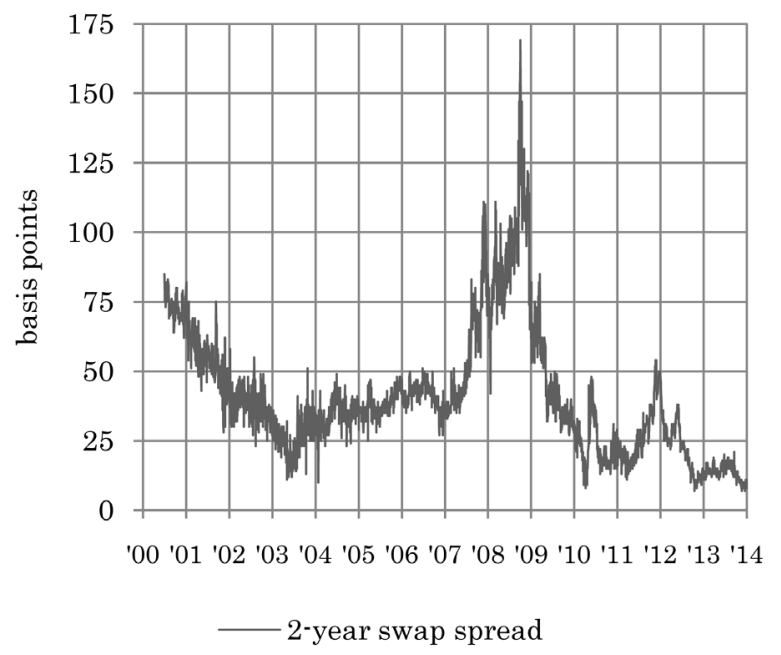

Figure 3. 2-year CMS vs. 2-year CMT.

Source: Federal Reserve Bank of St. Louis (FRED).

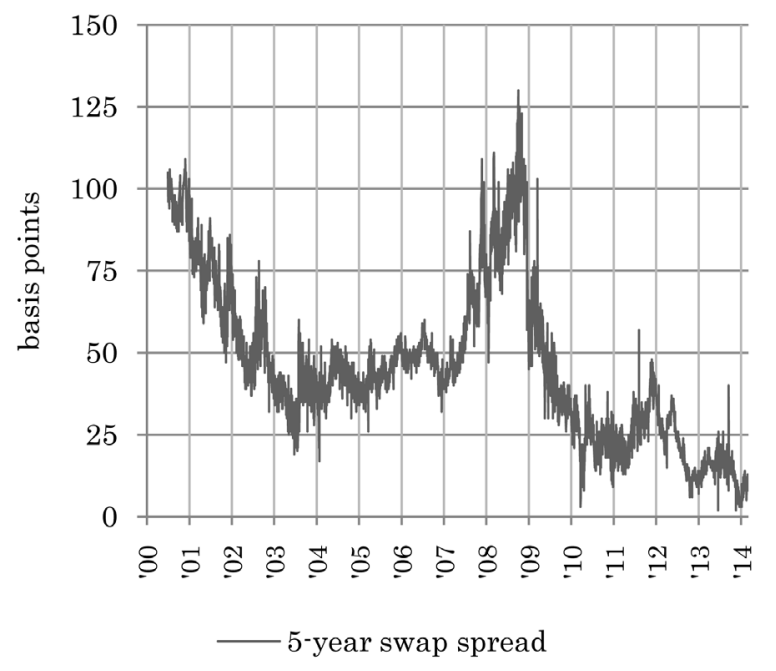

Figure 4. 5-year CMS vs. 5-year CMT.

Source: Federal Reserve Bank of St. Louis (FRED). 


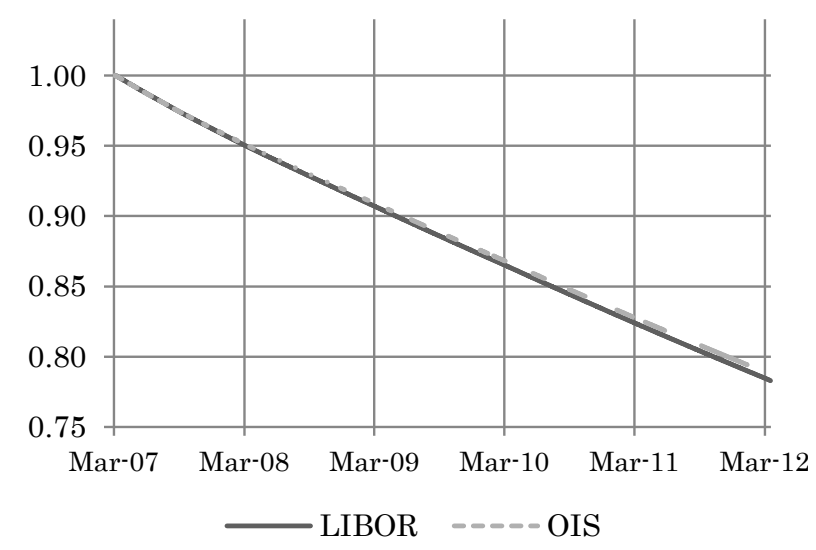

Figure 5. LIBOR and OIS discount factors as of March 15, 2007. Source: Bloomberg, L.P. (2014).

depicts, prior to the onset of the financial crisis, the difference between the LIBOR and OIS discount factors was negligible and the effect on pricing was immaterial.

As Figure 6 reveals, by the height of the financial crisis in October 2008, the spread between LIBOR and OIS discount factors had widened significantly. The widening occurred at all points of the term structure. As we will demonstrate in Section 13 of this paper, during this period of time, trades with short-dated tenors were equally susceptible to mispricing as their longer-dated brethren. Though the effects of the financial crisis had largely subsided by January 2012, the European sovereign debt crisis reignited fears in the credit markets and resulted in upward pressure on the spread between LIBOR and OIS. Figure 7 depicts the spread between the LIBOR and OIS discount factors as having contracted from its peak-crisis levels in the front end of the term structure. Further out the term structure, however, the spread persisted.

The spread between LIBOR and OIS reflects counterparty credit risk premium. Due to the mismatch in the tenor of the funding, the spread between LIBOR and OIS also reflects liquidity risk premium. The segmentation and dislocation (particularly noticeable in Figure 1 and Figure 2) that resulted from the financial crisis caused market participants to rethink the valuation methods that had been practiced for nearly 30 years.

As Figure 8 suggests, more recently, a non-negligible spread between the LIBOR and OIS discount factors persists.

Table 1 provides a summary of selected descriptive statistics on the spread between 3-month U.S. dollar LIBOR and 3-month U.S. dollar OIS in the pre-crisis, peak-crisis, post-crisis and recent market environments.

At this time, there is no evidence to suggest that the spread between LIBOR and OIS will return to the negligible levels observed prior to the financial crisis. Because LIBOR reflects credit risk and liquidity premium, it is an unreliable proxy for the risk-neutral discount rate, particularly during conditions of market stress.

\section{Intermezzo: The Credit Support Annex}

In 1985, the International Swaps and Derivatives Association (ISDA) developed the Master Agreement for parties to over-the-counter derivatives transactions. The 1985 Master Agreement contained standard definitions, representations and warranties, events of default and remedies. The purpose of the Master Agreement was to prevent disputes between counterparties and to aid in risk and credit management for the parties to a derivative transaction.

The Master Agreement lists collateralization through margining and close-out netting rules as the methods to mitigate counterparty risk. Collateralization provides the surviving party with recourse to the margined assets, which can be liquidated in the event of default. Collateralization also provides the surviving party with recourse to the value of the margined assets, which can be applied as assurance in the case of counterparty default. When default occurs, close-out netting rules typically apply. Multiple liabilities are netted into a single liability before the collateral is applied to the recovery. The Master Agreement defines the close-out amount as the amount of loss that the surviving party would incur to replace the economic equivalent of the pertinent transactions. The method by which the close-out amount is calculated is not well-defined by the Master Agreement. 


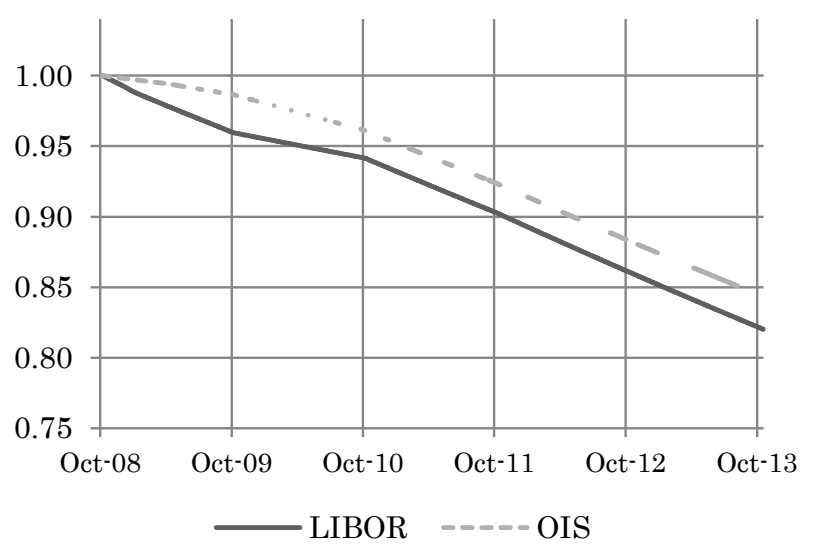

Figure 6. LIBOR and OIS discount factors as of October 10, 2008. Source: Bloomberg, L.P. (2014).

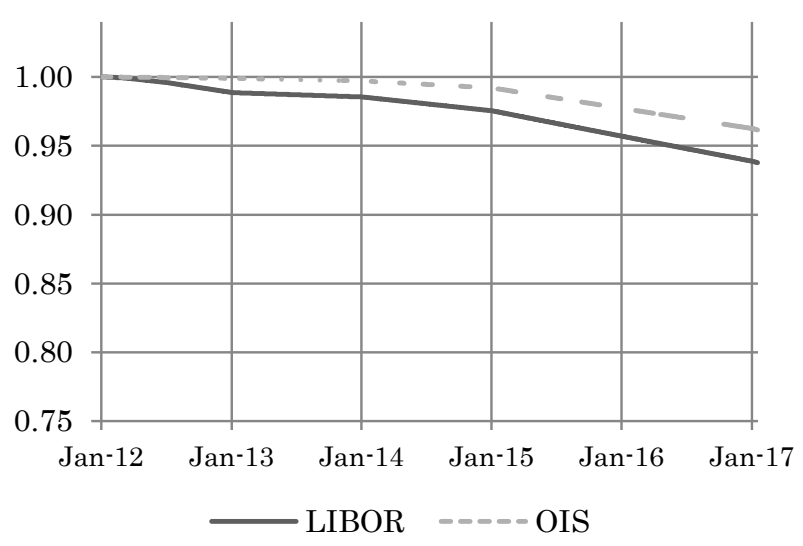

Figure 7. LIBOR and OIS discount factors as of January 4, 2012. Source: Bloomberg, L.P. (2014).

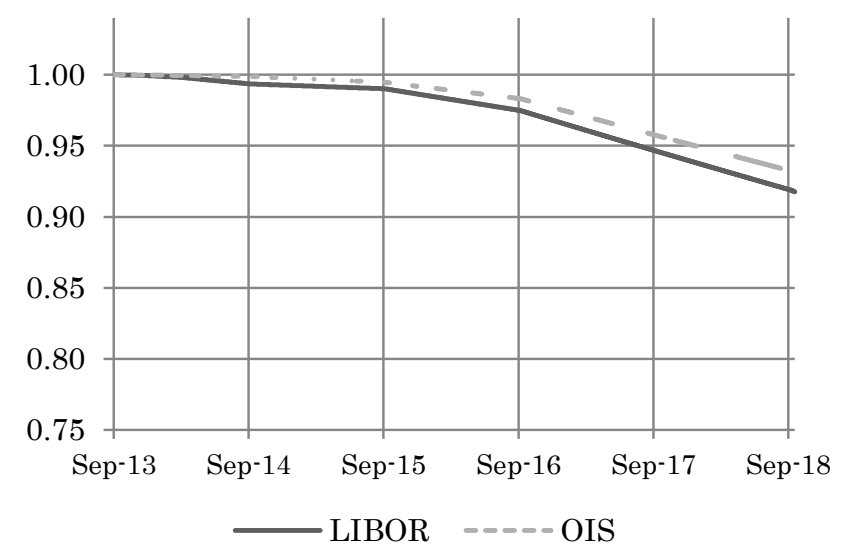

Figure 8. LIBOR and OIS discount factors as of Sept. 19, 2013. Source: Bloomberg, L.P. (2014).

In 1994, in response to demand for standardization, ISDA introduced the Credit Support Annex (CSA), which is an optional component of the Master Agreement. The CSA, which functions to mitigate credit risk between counterparties, requires the party with a negative economic exposure to post collateral with the opposing party to the transaction if the exposure to that party exceeds a certain previously negotiated threshold. By the mid-1990s, the practice of collateralization was widespread. In the wake of the financial crisis, posting collateral has be- 
Table 1. Descriptive statistics on the spread between 3-month U.S. Dollar LIBOR and 3month U.S. Dollar OIS.

\begin{tabular}{|c|c|c|c|c|c|}
\hline Time Period & Maximum & Minimum & Mean & Median & Std. Dev. \\
\hline Pre-Financial Crisis ${ }^{3}$ & 37.8 & 1.9 & 11.0 & 10.5 & 3.6 \\
\hline Financial Crisis of 2007-2008 & 364.4 & 10.3 & 84.5 & 73.5 & 58.7 \\
\hline European Debt Crisis ${ }^{5}$ & 50.1 & 12.1 & 30.2 & 30.3 & 9.5 \\
\hline calendar year 2013 & 17.6 & 12.9 & 15.5 & 15.4 & 0.9 \\
\hline
\end{tabular}

Source: Bloomberg, L.P. (2014)

come a standard practice as a means to mitigate the risk of counterparty default. The CSA has been ubiquitous since the financial crisis. Collateralization continues to be among the most widely-used practices to attenuate the counterparty risk in over-the-counter derivatives transactions.

Typically, the CSA agreement stipulates that collateral cash balances earn accrued interest. Interest is generally accrued on a daily basis using the interest rate and spread, if applicable, stipulated in the CSA agreement. Frequently, interest is calculated using an overnight rate (e.g. effective Federal Funds rate). The accrued interest is remunerated monthly to the party pledging the collateral, unless otherwise agreed between the parties. Under some agreements, interest may be compounded into the credit support balance instead of being remunerated each month.

The posting of collateral entails a cost for one party and a corresponding benefit for the other party. The receiver of the collateral eliminates or reduces its risk of loss conditional on default. In the event that no default occurs by the final maturity of the transactions, the party that had received the collateral repays the collateral plus any accrued interest to the party that had pledged the collateral. In the case of a default, after the collateral account is netted against the cash flows of the transactions, the collateral provider receives the remaining balance of the collateral, if any.

When permitted to do so per the terms of the CSA, the collateral receiver re-hypothecates the collateral for other purposes until the time at which the collateral must be returned to the provider. In the event that the collateral receiver defaults, it is possible that the provider of the collateral will receive only a fractional amount of the collateral provided. Under some agreements, the collateral receiver is not permitted to re-hypothecate the collateral. In these cases, the collateral must be placed in a segregated account.

CSA agreements are negotiated between parties. Agreements can include optionality, such as the choice of collateral type, the choice of currency (which can be valuable if there are significant movements in foreign exchange rates), initial margins, up threshold, down threshold and minimum transfer amounts-to name a few. The delivering party may also substitute collateral. Due to the embedded optionality, almost every CSA is unique. It has been said that "the CSA is the most exotic of exotic derivatives". Specific provisions of the CSA must be taken into account when pricing derivatives.

Before the financial crisis, the thresholds above which derivative counterparties were required to exchange collateral were often high or non-existent. In the aftermath of the financial crisis, thresholds were loweredsometimes to zero. It became standard practice for banks to demand daily collateral exchanges.

Setting thresholds at the portfolio level can result in non-linear effects on trade valuation. Non-zero thresholds reduce the amount of collateral received relative to the amount of collateral actually required to fund the future derivative cash flows. The manner in which this happens is discontinuous with regard to any particular trade in a given portfolio. For example, any trades executed while the threshold has not been breached will be uncollateralized. However, when the threshold is breached, collateral will be posted at the portfolio level and the transactions will be only partially collateralized. This discontinuous process results in a path-dependent change in funding, which results in a change in trade valuation. The magnitude of the non-linear effect is directly proportional to the size of the threshold; as the threshold increases, so, too, does the non-linear effect.

Trade valuation becomes even more complex if a threshold is also dependent on the credit rating of a given counterparty. The multi-factorial path dependencies complicate the modeling process. Respondents to the June 2013 ISDA Margin Survey (International Swaps and Derivatives Association, Inc., June 2013) indicate that greater than $90 \%$ of companies have existing CSA agreements containing thresholds that depend on credit rating. Nearly half of survey respondents are actively renegotiating CSA agreements in an effort to reduce or eliminate

${ }^{3}$ January 2002 to July 2007.

${ }^{4}$ August 2007 to September 2009.

${ }^{5}$ July 2011 to November 2012. 
thresholds that depend on credit ratings.

Given that the typical CSA stipulates that cash collateral earns interest at the relevant overnight rate, it follows that the OIS curve should be used for cash flow discounting. The currency of the collateral determines which OIS curve to use. If the CSA provides for optionality, then the cheapest-to-deliver (CTD) collateral should be used. Pursuant to most CSA agreements, cash is often that cheapest-to-deliver type of collateral in a low interest rate environment. For instance, a transaction that is collateralized by Euro cash would be discounted using the EONIA rate. A transaction that is collateralized under a CSA that permits delivery of collateral in a choice of currencies would be discounted using the OIS curve in the cheapest-to-deliver currency.

Prior to the onset of the financial crisis, banks were paying the Federal Funds rate on collateral and using the LIBOR curve to discount associated derivative contracts. When the crisis struck, banks were exposed to the basis between LIBOR and Federal Funds. They began discounting their derivatives using the OIS rate. Progressively, they shifted from term funding to overnight funding. Many CSA agreements stipulate the use of Overnight Index Swap rates as the funding rates for collateralized and centrally-cleared transactions.

The CSA takes the mark-to-market exposure of a portfolio of transactions in different currencies, nets them, and then requires collateral to cover the amount of economic exposure. Typically, the collateral is delivered in a single currency. Interest accrues at the overnight index rate corresponding to the relevant currency of the collateral delivered. This practice creates a mismatch between the funding currency and the amount of interest accrued between the underlying derivative cash flows and their associated collateral.

When a trade is collateralized, there are two separate sources of cash flows: contractual cash flows associated with the fixed versus floating legs of the swap and cash flows related to the collateral that is posted or received in order to secure the contractual cash flows. Since collateral typically earns the OIS rate, using the LIBOR curve to discount a transaction's cash flows is viewed by many market participants as problematic, as discussed in Section 9 of this paper.

In addition to the use of collateral in bilateral contracts, central clearing has become increasingly prominent in the derivatives marketplace. In order to mitigate counterparty risk, the global clearing houses require initial margin and variation margin to be posted against transactions that they clear. Collateral posted in this form typically earns interest computed by an overnight rate. The cost of funding the initial margin impacts the price of a given trade.

Given the complexities, organizations must have a clear collateral management policy that spans the entire lifecycle of the transaction - from pre-trade execution to daily collateral management.

We excerpt a few salient points from the June 2013 ISDA Margin Survey (International Swaps and Derivatives Association, Inc., 2013):

$\diamond$ Among all firms responding to the survey, $74 \%$ of all over-the counter derivatives trades (cleared and non-cleared) are subject to collateral agreements, compared with $29 \%$ in 2003.

$\diamond$ Responding firms reported that $69 \%$ of all non-cleared trades are subject to collateral agreements.

$\diamond$ The use of cash and government securities remains predominant; cash collateral represents $79 \%$ of the collateral delivered, while government securities represent $18 \%$ of the collateral delivered.

The use of collateral to mitigate counterparty credit risk resulting from over-the-counter derivative transactions is now ubiquitous.

\section{No-Arbitrage Arguments and the Law of One Price}

In March 2012, University of Toronto professors John Hull and Alan White published a controversial paper entitled "The Derivatives Discounting Dilemma” in the Journal of Investment Management (Hull, 2012). In doing so, they began the ongoing valuation debate that continues to this day. They assert that OIS rates should be used to price both collateralized trades and non-collateralized trades. They argue that the OIS curve is the best proxy for the "risk-free" rate for the purposes of applying risk-neutral valuation to calculate no-default derivative values and argue against the use of Funding Valuation Adjustment (FVA). Though they favor the use of OIS rates for the purposes of discounting, they reject the argument that it is appropriate to use OIS rates because the derivatives are funded by collateral and the interest rate most commonly paid on collateral is the effective Federal Funds rate. In doing so, they cite the long-established principle in finance that the evaluation of an investment should depend only on the risk of that investment, not the way in which it is funded. Further, they claim that the no-default value of a non-collateralized transaction should equal the no-default value of a collateralized transaction because in the absence of such equality between collateralized and non-collateralized valuations, there 
would be arbitrage. "To use LIBOR for non-collateralized transactions and OIS for collateralized transactions makes no sense and would seem to be a violation of the law of one price”, claim Hull and White (Hull, 2012).

Critics of Hull and White's approach posit that non-collateralized transactions should be funded at the firm's borrowing cost and that LIBOR is a reasonable estimate of this cost. Ceteris paribus, a non-collateralized transaction with a counter party that possesses a high credit rating should be worth more than a non-collateralized transaction with a counter party that possesses a comparatively lower credit rating. The curve used for discounting should be consistent with the way in which the transaction is collateralized or funded. Non-collateralized trades should be discounted using one's own cost of funding.

We share the prevailing view among market practitioners that various credit considerations such as Credit Valuation Adjustment (CVA), Debt Valuation Adjustment (DVA) and Funding Valuation Adjustment (FVA) must be taken into account when pricing and valuing derivative contracts. CVA and DVA cannot be modeled merely as the spread to a particular discounting curve. Funding Valuation Adjustment (FVA) cannot be considered as an additive term in the pricing because a derivative transaction's future cash flows depend on the funding decisions made at a future date. Pricing these cash flows today requires us to model funding decisions that will be made at some point in the future. Stated differently, the price of the derivative contract at time $t$ depends on one's funding strategy after time $t$. In turn, the funding strategy after time $t$ will depend on the derivative's price at times following time $t$. This recursive system is difficult to implement, particularly when payouts are path-dependent (Pallavicini, 2011).

Some practitioners argue that these adjustments must be made at the portfolio level, while others believe that the adjustments should be made at the trade level. There is an extensive literature on the various valuation adjustments such as CVA, FVA, DVA, Liquidity Valuation Adjustment (LVA) and Replacement Valuation Adjustment (RVA). The details of these adjustments can be quite involved and are beyond the scope of this paper. The reader is referred to Pallavicini et al. $(2011,2012)$ for additional details.

Prior to the onset of the financial crisis, many market practitioners used unilateral CVA to manage counterparty credit risk. This approach, which omits DVA, is justified when one of the two parties to the transaction can be considered default-free, as many banks were prior to 2007. In the aftermath of the financial crisis, however, we know that banks are susceptible to default. Under the assumption that both parties can default, the valuation applied to a particular trade will be asymmetric due to the differing default intensities, etc. applied to each party. This asymmetry is addressed by incorporating DVA, which results in a bilateral CVA.

In December 2011, the Bank for International Settlements indicated that "the most striking example of poor practice... was that some banks failed to account for the costs, benefits and risks of liquidity in all or some aspects of their business activities. These banks came to view funding liquidity as essentially free, and funding liquidity risk as essentially zero. As a result, there was simply no charge attributed to some assets for the cost of using funding liquidity, and conversely no credit attributed to some liabilities for the benefit of providing funding liquidity” (Bank for International Settlements, 2011).

Pricing derivative contracts involves considering all of the cash flows occurring when the transaction is initiated. These include the following:

a. the cash flows associated with the derivative transaction itself, along with the cash flows associated with the hedge instruments (i.e. replicating portfolio)

b. cash flows required to collateralize the derivative transaction and hedge instruments

c. cash flows required to fund the transactions

d. cash flows that occur in the event of default

Pricing is further complicated by the terms and conditions (e.g. optionality) of the CSA discussed in Section 8 of this paper, making it difficult for clients to compare price quotes among different banks. For example, even if three banks show the same all-in price, the economics may be quite different when one factors in the collateral costs/benefits. Valuation and collateral disputes can result from the variety of prices among counterparties for the same transaction. Additionally, the quotes from offshore banks may differ from those of local banks in that the offshore banks may include a sovereign credit risk premium in the CVA calculation.

This reinforces why the Law of One Price to clients with a non-standard CSA or without a CSA no longer applies. Trades are priced not only for their incremental market risk to a bank's portfolio, but also for their incremental counterparty, funding and capital implications to that bank's portfolio. The marginal contribution of a new trade could either increase or decrease a bank's counterparty and funding risks. The same principle holds true for pre-existing trades that are unwound. 


\section{Banks Capitalize on an Opportunity}

In this section, we reveal how a few large banks earned billions of dollars in profit by switching from LIBOR to OIS discounting and we provide guidance to market participants when pricing, amending or unwinding transactions.

Even before the onset of the financial crisis, a few large, market-making banks realized the benefit of using the OIS curve for pricing trades collateralized by cash. In doing so, these investment banks earned billions of dollars in profit by switching from LIBOR to OIS discounting. They built systems to handle the intricacies of this new methodology and employed teams of lawyers to analyze the details of their CSA agreements. These banks also spent a considerable amount of time and money analyzing the $\mathrm{P} \& \mathrm{~L}$ effects of switching from LIBOR to OIS discounting. They then optimized and prepositioned their portfolios to benefit from the switch and traded on the information they gathered.

The banks' strategy to monetize the difference between the two discounting methodologies was simple. Using the lower OIS rates to discount a trade resulted in a greater liability than when discounted using LIBOR rates. A bank with a trade that was significantly out-of-the-money could pay its counterparty an above-market termination fee in exchange for unwinding the trade because the majority of market participants was still using LIBOR for discounting purposes. The termination would still be profitable because the bank had paid less than the present value of the trade when discounted on the OIS curve.

For example, the present value of a 3-year swap might be $\$ 75$ million when discounted on the LIBOR curve. The same transaction might have a present value of $\$ 78$ million when discounted on the OIS curve. The bank would, for instance, pay its counterparty $\$ 76$ million to unwind the trade-an amount greater than its competitors would pay in order to step into the trade upon assignment/ novation. This practice would result in a profit for the bank of $\$ 2$ million in this example.

Goldman Sachs is believed to have identified of appropriateness of OIS discounting in the early 2000s. They switched to this new valuation technique some time in 2008, two years before many of its competitors. According to its annual reports, Goldman Sachs received cash collateral of \$137 billion in 2008, an increase of $132 \%$ on the $\$ 59$ billion in collateral that it received in 2007. Meanwhile, the amount of cash collateral it posted in 2008 was $\$ 34$ billion, a mere 22\% increase relative to the \$27 billion it posted in 2007. Goldman’s derivative assets increased massively in size, while its derivatives liabilities barely changed by comparison (Cameron, 2013).

Most of the early arbitrage activity occurred in the interest rate derivatives markets, but later migrated to other markets such as currency and commodity derivatives, where most participants were unaware of the implications of CSA discounting.

Certain savvy end-user clients took advantage of the differential in discounting between different banks. These end-users were able to recognize significant value by transacting with those banks who were offering more advantageous pricing than competitor banks, who were mispricing trades by continuing to use the LIBOR curve for pricing. For example, clients were able to profit by assigning trades to third parties instead of unwinding these trades with existing counterparties.

\section{Introduction of the Standard Credit Support Annex}

Given the variability in pricing that results from incorporating a client's counterparty credit and funding costs, most banks are focused on standardizing the CSA. In June 2013, ISDA published the Standard Credit Support Annex (SCSA). The purpose of the SCSA is to standardize existing market practices in collateral management for OTC derivatives by removing any optionality embedded in the existing CSA and promoting the use of OIS discounting. According to ISDA, "The SCSA retains the operational mechanics of the current CSA, but amends the collateral calculation so that derivative exposures and offsetting collateral are grouped into like currencies, or "silos". The SCSA contemplates the sole use of cash as eligible collateral for variation margin..." The SCSA will still permit the use of securities for initial margin. Each of the silos will be valued independently in order to generate the amount of collateral to be transferred in the given currency. This new method is consistent with the margin approaches that have been instituted by the various clearing houses globally.

The SCSA removes the optionality embedded in the existing CSA. This is achieved by allocating every transaction to one of 17 currency "silos" based on the currency of the underlying transaction. Counterparties will only be allowed to post cash collateral in that currency and the trades in each silo will be discounted using the relevant OIS curve (or an agreed upon alternative if a liquid OIS curve does not exist). For example, a trade denominated in U.S. dollars would be allocated to the U.S. dollar silo, with counterparties posting U.S. dollar cash 
collateral and discounting trades in the U.S. dollar silo accordingly, using the U.S. dollar OIS curve.

While the silo structure eliminates problems caused by optionality in the existing CSA, it creates a new issue: cross-currency settlement risk. The existence of 17 silos means that counterparties could post 17 separate currencies back and forth each day, resulting in cross-currency settlement risk (or "Herstatt risk"). To mitigate this risk, ISDA has devised the Implied Swap Rate (ISR) methodology. This methodology allows each counterparty to aggregate the various collateral cash flows into a single payment in one of 7 "transport" currencies. Counterparties allocate their various trades to the silos and calculate valuations using the relevant OIS curve. They then settle in a single currency, thus eliminating the cross-currency settlement risk.

\section{The Switch to OIS Discounting}

We noted earlier that Goldman Sachs switched to OIS discounting in 2008. A few of its competitors switched to OIS discounting in 2010. For instance, in the fourth quarter of 2010, Morgan Stanley changed its valuation methodology and adopted OIS discounting for all of its collateralized interest rate derivative contracts. Morgan Stanley issued a statement to the Securities and Exchange Commission noting that “... all collateralized derivatives products will move to OIS discounting over time, but the use of OIS is most strongly evident in the interest rate derivatives market at this time" (Securities and Exchange Commission, 2011). In June 2010, LCH. Clearnet Group Ltd., which clears approximately $50 \%$ of the global interest rate swaps market, changed the margining basis for its \$218 trillion Swap Clear portfolio of vanilla USD, EUR and GBP interest rate swaps from LIBOR to OIS.

The June 2013 ISDA Margin Survey (International Swaps and Derivatives Association, Inc., June 2013) results indicate that $70 \%$ of total respondents had implemented either OIS discounting or CSA-specific discounting, while only $30 \%$ of total respondents continued to use the LIBOR curve for valuation. Curiously, the Survey results indicate that market participants in the commodities space have been slower to embrace the OIS discounting methodology, with only $30 \%$ of respondents having implemented the OIS for valuation. The remaining $70 \%$ of respondents in the commodities space continue to use the LIBOR curve for the purposes of discounting. In 2008, The Wall Street Journal published a series of articles which raised questions about the legitimacy of the LIBOR rates submitted by certain contributor banks. An article in April 2008 (Mollencamp, 2008) claimed that LIBOR “ . .. is becoming unreliable”. In the months that followed, it was revealed that certain contributor banks understated the rates they were paying to secure short-term financing because they did not want to raise concerns among market participants that they might be desperate for cash amid the financial crisis. Moreover, it has been alleged that certain contributor banks manipulated the LIBOR fixings in an effort to enhance the profitability of their derivatives portfolios. Contrary to widely-held belief, the impetus for market participants' migrating from LIBOR to OIS discounting was not the alleged manipulation in the rate setting process, but rather the notion that a collateral account is typically remunerated at an overnight interest rate per the terms of the CSA agreement. The alleged manipulation in the rate setting process merely reinforced the appropriateness of using OIS rates.

Until there is more consistency among asset classes (see Figure 9), many corporations will continue to use the LIBOR curve for valuation purposes. If the spread between LIBOR and OIS were to widen, these firms

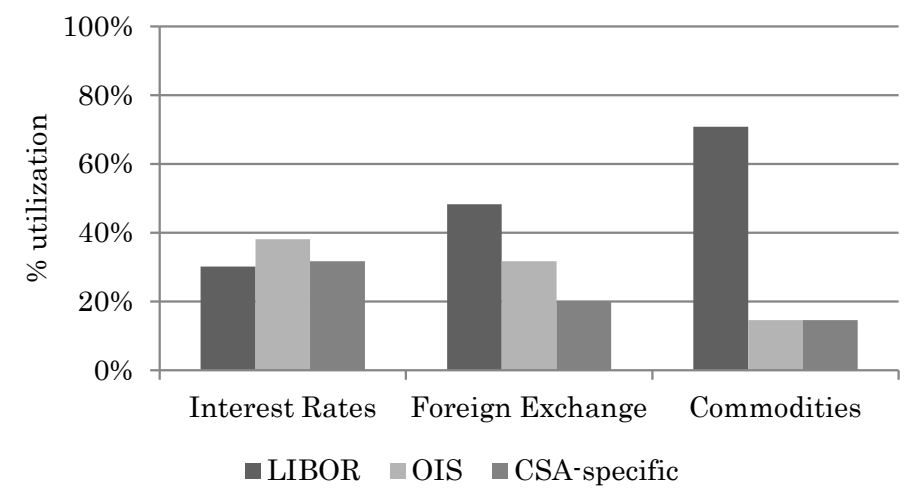

Figure 9. Discounting methodologies by asset class. Source: June 2013 ISDA Margin Survey. 
would be required by the Dodd-Frank Act to reconcile valuation differences and to report these differences to regulators.

\section{Case Studies}

\subsection{Methods}

In the case studies that follow, we demonstrate the impact of CSA discounting on pricing, valuation and risk management in the pre-crisis, peak-crisis and post-crisis and recent market environments. We assume the presence of a bilateral, zero-threshold CSA agreement, which stipulates OIS as the rate of interest paid on the collateral. In each market environment, we examine two transactions in isolation: a long position in a crude oil swap and a long position in an Asian cap (i.e. a call option with an Asian-style payoff) on crude oil. The underlying for each transaction is NYMEX West Texas Intermediate (WTI) crude oil ${ }^{6}$. We further assume that the Asian caps have a delta of 30 percent at initiation.

We price the options using a two-factor, relative mean-reverting model (Gabillion, 1991), early-expiration volatilities, and an approach similar to Turnbull \& Wakeman (Turnbull, 1991), where we apply moment matching to determine the distribution of the average. We compute the exact average forward price of NYMEX WTI and the exact variance of the average (assuming that the underlying price process of NYMEX WTI is lognormal). We then assume that the lognormal distribution of the average has these two values for the first and second central moments. These quantities, along with the options' strike and maturity, are then input to a European Black-Scholes calculator.

We shift the NYMEX WTI forward curve by a total of $\pm 25 \%$ from the base forward curve in increments $5 \%$. We re-compute the present value, denoted by PV, of trades at each successively higher or lower forward price using both LIBOR discounting and OIS discounting. We compute the first-order Greek sensitivities delta, vega, theta and rho, as well as the second-order Greek sensitivity gamma by bumping the underlying market variables. Tables 6-9 display the pricing and risk results by attribute for the Asian cap in the various market environments under each discounting method.

While holding the forward prices constant, we then shift the NYMEX WTI implied volatilities by a total of $\pm 15 \%$ in absolute terms (i.e. volatility points) from the base implied volatilities in increments $3 \%$. We re-compute the present value, denoted by PV, of each of the trades at each incremental higher or lower forward price using both LIBOR discounting and OIS discounting. We compute the first-order Greek sensitivities delta, vega, theta and rho, as well as the second-order Greek sensitivity gamma by bumping the underlying market variables. Tables 6-9 displays the pricing and risk results by attribute for the Asian cap in the various market environments under each discounting method.

\subsection{Risk Sensitivities}

The first-order Greek sensitivity delta is computed as

$$
\Delta=\frac{\partial V}{\partial S}=\frac{\text { central difference }}{\text { bump size } \cdot 2}
$$

The second-order Greek sensitivity gamma is computed as

$$
\Gamma=\frac{\partial \Delta}{\partial S}=\frac{\partial^{2} V}{\partial S^{2}}=\frac{\mathrm{d} \Delta}{\left(\frac{\mathrm{d} S}{S}\right)} \cdot(0.01) \Rightarrow \Gamma \frac{S}{100}
$$

where

$$
\Gamma=\frac{\mathrm{d} \Delta}{\mathrm{d} S}
$$

Which is computed as

$$
\Gamma=\frac{\text { second difference }}{(\text { bump size })^{2}}=\frac{S(K+n)-2 S(K)-S(K-n)}{n^{2}}
$$

\footnotetext{
${ }^{6}$ Although we use crude oil in our analysis, the concepts here are easily extended to other commodities such as natural gas, refined products, metals, agriculture, etc.
} 
The delta and gamma calculations include the effects of volatility skew. Specifically, the options were priced using a "sticky-delta" method. Under the sticky-delta method, the volatility smile depends only on delta. An option's delta is a function of its strike and underlying asset price, inter alia. Therefore, the volatility smile depends on the strike of the option and the price of the underlying. As a result, the volatility used for pricing an option of a given strike will change if the price of the underlying changes.

The delta calculation will include an additional contribution from the Greek vega weighted by the sensitivity of the volatility of the underlying price. Stated differently, under the sticky-delta method, the Greek delta measures the total sensitivity of the option's price with respect to the price of the underlying. Thus, this Greek calculation method is commonly referred to as "skew-adjusted".

For example, let us consider an out-of-the money cap that is priced with an implied volatility of $30 \%$. If the forward price of the underlying is bumped up, then the option will move closer to at-the-money. As a result, the volatility used to price this option is no longer $30 \%$, but typically something less than $30 \%$ due to the presence of a volatility skew. In this example, the value of delta is less than what the standard Black-Scholes delta calculation would produce because the volatility is held constant in the latter calculation, despite the existence of a volatility skew.

The first-order Greek sensitivity vega is computed as:

$$
v=\frac{\partial V}{\partial \sigma} \cdot(0.01)=\frac{\text { central difference }}{\text { volatility bump size } \cdot 2}
$$

The first-order Greek sensitivity theta, denoted by $=-\frac{\partial V}{\partial \tau}$, is computed by moving each of the four "as of" dates in our analysis forward by one day and holding constant the forward prices relative to their specific absolute dates on all market data curves and surfaces.

The first-order Greek sensitivity rho, $\frac{\partial V}{\partial r}$, is computed by bumping the continuously compounded short interest rates (zero-coupon rates), or equivalently the discount factors used to compute them. The continuously compounded spot interest rates and the discount factors are related by:

$$
\mathrm{d} f\left(t_{0}, T\right)=\exp \left[-r\left(t_{0}, T\right) T\right]
$$

where $\mathrm{d} f\left(t_{0}, T\right)$ is the discount factor from time $t_{0}$ to the realization of the cash flow and $r\left(t_{0}, T\right)$ is the spot interest rate from time $t_{0}$ to maturity date $T$. Bumping the spot interest rates by $\Delta r$ (e.g. one basis point) is equivalent to bumping the discount factor by:

$$
\mathrm{d} f^{\prime}\left(t_{0}, T\right)=\mathrm{d} f\left(t_{0}, T\right) \exp [-\Delta r T]
$$

\subsection{Trade Parameters and Relevant Market Data}

The Tables 2-5 summarize the trade parameters in each of the market environments included in our analysis.

\begin{tabular}{ccc} 
Table 2. Pre-Crisis (as of March 15, 2007). & \\
\hline & Swap & Asian Cap \\
\hline Start Date & 01 -Apr-2007 & $01-$ Apr-2007 \\
End Date & 31 -Mar-2010 & 31 -Mar-2010 \\
Volume (monthly) & 50,000 barrels & 50,000 barrels \\
Price/Strike & $\$ 65.10$ & $\$ 75.65$ \\
Underlying & NYMEX WTI & NYMEX WTI \\
Pricing Convention & Daily average & Daily average \\
Present Value $(t=0)$ & $\$ 0$ & $\$ 0$
\end{tabular}

\footnotetext{
${ }^{7}$ The strike prices of the Asian caps were calibrated such that the trades' deltas were $30 \%$ at initiation.
} 
Table 3. Peak-Crisis (as of October 10, 2008).

\begin{tabular}{ccc}
\hline & Swap & Asian Cap \\
\hline Start Date & 01-Nov-2008 & 01-Nov-2008 \\
End Date & 31 -Oct-2011 & 31 -Oct-2011 \\
Volume (monthly) & 50,000 barrels & 50,000 barrels \\
Price/Strike & $\$ 84.68$ & $\$ 110.07$ \\
Underlying & NYMEX WTI & NYMEX WTI \\
Pricing Convention & Daily average & Daily average \\
Present Value $(t=0)$ & $\$ 0$ & $\$ 0$ \\
\hline
\end{tabular}

Table 4. Post-Crisis (as of January 4, 2012).

\begin{tabular}{ccc}
\hline & Swap & Asian Cap \\
\hline Start Date & 01-Feb-2012 & 01-Feb-2012 \\
End Date & 31-Jan-2015 & 31-Jan-2015 \\
Volume (monthly) & 50,000 barrels & 50,000 barrels \\
Price/Strike & $\$ 123.75$ \\
Underlying & NY9.31 & NYMEX WTI \\
Pricing Convention & Daily average & Daily average \\
Present Value $(t=0)$ & $\$ 0$ & $\$ 0$ \\
\hline
\end{tabular}

Table 5. Recent (as of September 13, 2013).

\begin{tabular}{ccc}
\hline & Swap & Asian Cap \\
\hline Start Date & 01-Oct-2013 & 01-Oct-2013 \\
End Date & 30-Sep-2016 & 30-Sep-2016 \\
Volume (monthly) & 50,000 barrels & 50,000 barrels \\
Price/Strike & \$104.97 \\
Underlying & NYMEX WTI & NYMEX WTI \\
Pricing Convention & Daily average & Daily average \\
Present Value $(t=0)$ & $\$ 0$ & \$0
\end{tabular}

Figure 10 depicts the forward price curves for NYMEX WTI in the pre-crisis, peak-crisis, post-crisis and recent market environments. The term structure of oil moves between states of contango and backwardation over time.

Figure 11 depicts the at-the-money (ATM) implied volatilities for NYMEX WTI in the pre-crisis, peak-crisis, post-crisis and recent market environments.

\subsection{Pricing and Risk Computations}

Table 6 displays the pricing and risk results by attribute for the Asian cap as of March 15, 2007 under each discounting method, with forward prices and implied volatilities shifted a total of $\pm 25 \%$ and $\pm 15 \%$, respectively.

Table 7 displays the pricing and risk results by attribute for the Asian cap as of October 10, 2008 under each discounting method, with forward prices and implied volatilities shifted a total of $\pm 25 \%$ and $\pm 15 \%$, respectively. 
Table 6. Pre-Crisis (as of March 15, 2007).

\begin{tabular}{|c|c|c|c|c|c|c|c|c|}
\hline \multirow[b]{2}{*}{ Attribute } & \multicolumn{4}{|c|}{ Forward Prices } & \multicolumn{4}{|c|}{ Implied Volatilities } \\
\hline & Money-ness ${ }^{\dagger}$ & LIBOR & OIS & $\%$ Variation & Money-ness $^{\dagger+}$ & LIBOR & OIS & \% Variation \\
\hline PV & & $13,777,938$ & $13,800,717$ & $0.2 \%$ & & $5,244,861$ & $5,255,271$ & $0.2 \%$ \\
\hline$\Delta$ & & $1,150,899$ & $1,152,906$ & $0.2 \%$ & & 694,463 & 695,745 & $0.2 \%$ \\
\hline$\Gamma$ & $25 \%$ & 14,023 & 14,042 & $0.1 \%$ & $12 \%$ & 9571 & 9585 & $0.1 \%$ \\
\hline$v$ & & 595,019 & 596,231 & $0.2 \%$ & & 516,839 & 517,903 & $0.2 \%$ \\
\hline$\Theta$ & & $(16,525)$ & $(16,507)$ & $(0.1 \%)$ & & $(18,081)$ & $(18,083)$ & $0.0 \%$ \\
\hline$\rho$ & & (3147) & (3155) & $0.3 \%$ & & (2015) & (2021) & $0.3 \%$ \\
\hline PV & & $4,337,134$ & $4,345,173$ & $0.2 \%$ & & $2,054,021$ & $2,058,153$ & $0.2 \%$ \\
\hline$\Delta$ & & 783,192 & 784,559 & $0.2 \%$ & & 611,744 & 613,095 & $0.2 \%$ \\
\hline$\Gamma$ & $10 \%$ & 14,758 & 14,779 & $0.1 \%$ & $6 \%$ & 11,187 & 11,209 & $0.2 \%$ \\
\hline$v$ & & 575,235 & 576,410 & $0.2 \%$ & & 503,784 & 504,826 & $0.2 \%$ \\
\hline$\Theta$ & & $(14,086)$ & $(14,085)$ & $0.0 \%$ & & $(13,315)$ & $(13,318)$ & $0.0 \%$ \\
\hline$\rho$ & & (1815) & (1821) & $0.3 \%$ & & (1462) & (1466) & $0.3 \%$ \\
\hline PV & & 0 & 0 & - & & 0 & 0 & - \\
\hline$\Delta$ & & 540,123 & 541,161 & $0.2 \%$ & & 540,123 & 541,161 & $0.2 \%$ \\
\hline$\Gamma$ & $0 \%$ & 12,464 & 12,483 & $0.2 \%$ & $0 \%$ & 12,464 & 12,483 & $0.2 \%$ \\
\hline$v$ & & 485,438 & 486,451 & $0.2 \%$ & & 485,438 & 486,451 & $0.2 \%$ \\
\hline$\Theta$ & & $(10,176)$ & $(10,179)$ & $0.0 \%$ & & $(10,176)$ & $(10,179)$ & $0.0 \%$ \\
\hline$\rho$ & & (1098) & (1102) & $0.4 \%$ & & (1098) & (1102) & $0.4 \%$ \\
\hline PV & & $(2,748,483)$ & $(2,755,199)$ & $0.2 \%$ & & $(1,909,742)$ & $(1,914,229)$ & $0.2 \%$ \\
\hline$\Delta$ & & 303,525 & 304,156 & $0.2 \%$ & & 435,983 & 436,900 & $0.2 \%$ \\
\hline$\Gamma$ & $(10 \%)$ & 8130 & 8146 & $0.2 \%$ & (6\%) & 13,422 & 13,447 & $0.2 \%$ \\
\hline$v$ & & 326,468 & 327,202 & $0.2 \%$ & & 442,185 & 443,173 & $0.2 \%$ \\
\hline$\Theta$ & & (6252) & (6257) & $0.1 \%$ & & (7089) & (7094) & $0.1 \%$ \\
\hline$\rho$ & & (515) & (517) & $0.4 \%$ & & (709) & (711) & $0.3 \%$ \\
\hline PV & & $(4,690,290)$ & $(4,701,188)$ & $0.2 \%$ & & $(4,224,009)$ & $(4,234,218)$ & $0.2 \%$ \\
\hline$\Delta$ & & 117,291 & 117,540 & $0.2 \%$ & & 212,035 & 212,443 & $0.2 \%$ \\
\hline$\Gamma$ & (25\%) & 3731 & 3739 & $0.2 \%$ & $(12 \%)$ & 11,305 & 11,327 & $0.2 \%$ \\
\hline$v$ & & 144,181 & 144,509 & $0.2 \%$ & & 291,952 & 292,580 & $0.2 \%$ \\
\hline$\Theta$ & & (2486) & (2489) & $0.1 \%$ & & (2818) & $(2,821)$ & $0.1 \%$ \\
\hline$\rho$ & & (156) & (156) & $0.0 \%$ & & (216) & (217) & $0.5 \%$ \\
\hline
\end{tabular}

† refers to the money-ness of the NYMEX WTI forward prices, not the delta of the Asian caps; †† refers to the money-ness of the NYMEX WTI implied volatilities, not the delta of the Asian caps. 
Table 7. Peak-Crisis (as of October 10, 2008).

\begin{tabular}{|c|c|c|c|c|c|c|c|c|}
\hline \multirow[b]{2}{*}{ Attribute } & \multicolumn{4}{|c|}{ Forward Prices } & \multicolumn{4}{|c|}{ Implied Volatilities } \\
\hline & Money-ness ${ }^{\dagger}$ & LIBOR & OIS & $\%$ Variation & Money-ness ${ }^{\dagger \dagger}$ & LIBOR & OIS & \% Variation \\
\hline PV & & $15,305,525$ & $15,655,868$ & $2.3 \%$ & & $4,636,990$ & $4,740,677$ & $2.2 \%$ \\
\hline$\Delta$ & & 899,233 & 919,929 & $2.3 \%$ & & 637,037 & 650,851 & $2.2 \%$ \\
\hline$\Gamma$ & $25 \%$ & 9577 & 9779 & $2.1 \%$ & $12 \%$ & 6935 & 7081 & $2.1 \%$ \\
\hline$v$ & & 880,913 & 900,728 & $2.2 \%$ & & 677,534 & 692,662 & $2.2 \%$ \\
\hline$\Theta$ & & $(44,158)$ & $(44,947)$ & $1.8 \%$ & & $(36,205)$ & $(36,870)$ & $1.8 \%$ \\
\hline$\rho$ & & $(4721)$ & (4905) & $3.9 \%$ & & (2824) & (2924) & $3.5 \%$ \\
\hline PV & & $5,199,174$ & $5,313,840$ & $2.2 \%$ & & $1,820,015$ & $1,860,718$ & $2.2 \%$ \\
\hline$\Delta$ & & 679,552 & 694,533 & $2.2 \%$ & & 579,749 & 592,768 & $2.2 \%$ \\
\hline$\Gamma$ & $10 \%$ & 8660 & 8849 & $2.2 \%$ & $6 \%$ & 7362 & 7529 & $2.3 \%$ \\
\hline$v$ & & 756,551 & 773,577 & $2.3 \%$ & & 659,006 & 674,064 & $2.3 \%$ \\
\hline$\Theta$ & & $(34,055)$ & $(34,685)$ & $1.8 \%$ & & $(30,457)$ & $(31,040)$ & $1.9 \%$ \\
\hline$\rho$ & & (2913) & (3015) & $3.5 \%$ & & (2332) & (2415) & $3.6 \%$ \\
\hline PV & & 0 & 0 & - & & 0 & 0 & - \\
\hline$\Delta$ & & 537,267 & 549,621 & $2.3 \%$ & & 537,267 & 549,621 & $2.3 \%$ \\
\hline$\Gamma$ & $0 \%$ & 7620 & 7802 & $2.4 \%$ & $0 \%$ & 7620 & 7802 & $2.4 \%$ \\
\hline$v$ & & 641,750 & 656,618 & $2.3 \%$ & & 641,750 & 656,618 & $2.3 \%$ \\
\hline$\Theta$ & & $(26,615)$ & $(27,140)$ & $2.0 \%$ & & $(26,615)$ & $(27,140)$ & $2.0 \%$ \\
\hline$\rho$ & & (2011) & (2082) & $3.5 \%$ & & (2011) & (2082) & $3.5 \%$ \\
\hline PV & & $(4,027,654)$ & $(4,115,278)$ & $2.2 \%$ & & $(1,759,433)$ & $(1,798,687)$ & $2.2 \%$ \\
\hline$\Delta$ & & 404,968 & 413,877 & $2.2 \%$ & & 492,659 & 503,283 & $2.2 \%$ \\
\hline$\Gamma$ & $(10 \%)$ & 6381 & 6524 & $2.2 \%$ & (6\%) & 7879 & 8050 & $2.2 \%$ \\
\hline$v$ & & 511,411 & 522,908 & $2.2 \%$ & & 620,789 & 634,572 & $2.2 \%$ \\
\hline$\Theta$ & & $(19,522)$ & $(19,908)$ & $2.0 \%$ & & $(22,861)$ & $(23,293)$ & $1.9 \%$ \\
\hline$\rho$ & & (1308) & (1353) & $3.4 \%$ & & (1702) & (1759) & $3.3 \%$ \\
\hline PV & & $(8,022,893)$ & $(8,199,678)$ & $2.2 \%$ & & $(4,255,411)$ & $(4,349,629)$ & $2.2 \%$ \\
\hline$\Delta$ & & 226,535 & 230,692 & $1.8 \%$ & & 418,714 & 427,784 & $2.2 \%$ \\
\hline$\Gamma$ & (25\%) & 3990 & 4059 & $1.7 \%$ & $(12 \%)$ & 8194 & 8375 & $2.2 \%$ \\
\hline$v$ & & 302,070 & 307,852 & $1.9 \%$ & & 578,092 & 590,961 & $2.2 \%$ \\
\hline$\Theta$ & & $(10,308)$ & $(10,502)$ & $1.9 \%$ & & $(17,427)$ & $(17,765)$ & $1.9 \%$ \\
\hline$\rho$ & & (572) & (587) & $2.6 \%$ & & (1262) & (1303) & $3.2 \%$ \\
\hline
\end{tabular}

$\dagger$ refers to the money-ness of the NYMEX WTI forward prices, not the delta of the Asian caps; †† refers to the money-ness of the NYMEX WTI implied volatilities, not the delta of the Asian caps. 


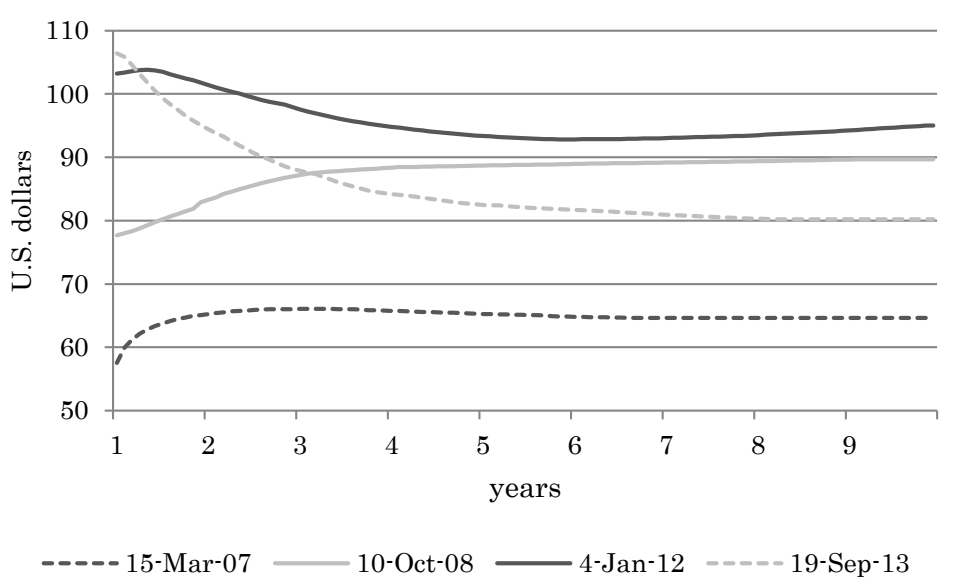

Figure 10. Forward price curves for NYMEX WTI in each market environment. Source: Bloomberg, L.P. (2014).

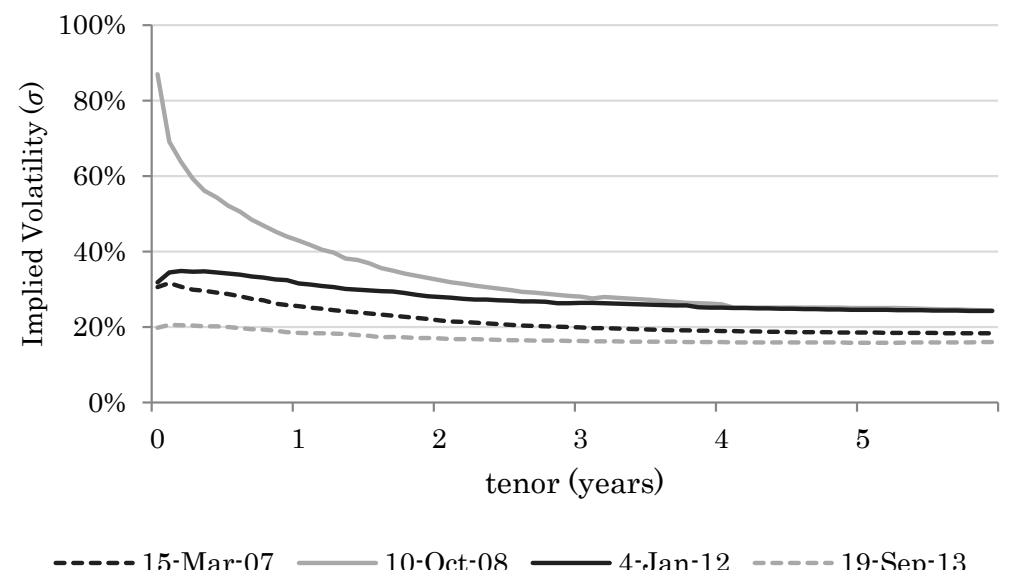

Figure 11. At-the-money (ATM) implied volatilities for NYMEX WTI in each market environment. Source: Bloomberg, L.P. (2014).

Table 8 displays the pricing and risk results by attribute for the Asian cap as of January 4, 2012 under each discounting method, with forward prices and implied volatilities shifted a total of $\pm 25 \%$ and $\pm 15 \%$, respectively.

Table 9 displays the pricing and risk results by attribute for the Asian cap as of September 19, 2013 under each discounting method, with forward prices and implied volatilities shifted a total of $\pm 25 \%$ and $\pm 15 \%$, respectively.

In the interest of brevity, the pricing results for the $\pm 5 \%, \pm 15 \%$ and $\pm 20 \%$ shifts in forward prices have been omitted from Tables $6-9$. The pricing results for the $\pm 3 \%, \pm 9 \%$ and $\pm 12 \%$ shifts in implied volatilities have also been omitted from Tables 6-9. Full details are available from the author upon written request.

\subsection{Impact on Valuation}

Figure 12 plots the monetary difference in the swaps' present value computed under LIBOR discounting and OIS discounting as the forward prices of NYMEX WTI shift in $\pm 5 \%$ increments.

The swaps' value is zero at initiation under both discounting methodologies. The impact on valuation is linear as the forward prices of NYMEX WTI move up and down. The impact becomes particularly noticeable as the trades' present value moves significantly in-the-money or out-of-the-money. During the height of the financial crisis in October 2008, the trades' present value would be impacted materially by $\pm \$ 800,000$ if the forward prices of NYMEX WTI were to move $\pm 25 \%$.

As Figure 13 depicts, the impact on valuation of the Asian cap closely mirrors that of a swap, although in non-linear fashion, when forward prices are shocked by a total of $\pm 25 \%$ in increments of $5 \%$. 
Table 8. Post-Crisis (as of January 4, 2012).

\begin{tabular}{|c|c|c|c|c|c|c|c|c|}
\hline \multirow[b]{2}{*}{ Attribute } & \multicolumn{4}{|c|}{ Forward Prices } & \multicolumn{4}{|c|}{ Implied Volatilities } \\
\hline & Money-ness ${ }^{\dagger}$ & LIBOR & OIS & $\%$ Variation & Money-ness ${ }^{\dagger \dagger}$ & LIBOR & OIS & $\%$ Variation \\
\hline PV & & $21,500,450$ & $21,725,898$ & $1.0 \%$ & & $10,034,782$ & $10,167,539$ & $1.3 \%$ \\
\hline$\Delta$ & & $1,169,802$ & $1,175,899$ & $0.5 \%$ & & 750,619 & 762,146 & $1.5 \%$ \\
\hline$\Gamma$ & $25 \%$ & 12,848 & 12,934 & $0.7 \%$ & $12 \%$ & 9216 & 9337 & $1.3 \%$ \\
\hline$v$ & & $1,058,798$ & $1,069,961$ & $1.1 \%$ & & 868,454 & 883,116 & $1.7 \%$ \\
\hline$\Theta$ & & $(36,802)$ & $(37,638)$ & $2.3 \%$ & & $(38,859)$ & $(39,669)$ & $2.1 \%$ \\
\hline$\rho$ & & (5315) & (5407) & $1.7 \%$ & & (3880) & (4054) & $4.5 \%$ \\
\hline PV & & $6,558,185$ & $6,645,312$ & $1.3 \%$ & & $3,876,868$ & 3,928,198 & $1.3 \%$ \\
\hline$\Delta$ & & 790,107 & 799,438 & $1.2 \%$ & & 637,505 & 646,227 & $1.4 \%$ \\
\hline$\Gamma$ & $10 \%$ & 14,303 & 14,431 & $0.9 \%$ & $6 \%$ & 10,636 & 10,775 & $1.3 \%$ \\
\hline$v$ & & 969,192 & 981,080 & $1.2 \%$ & & 830,290 & 841,868 & $1.4 \%$ \\
\hline$\Theta$ & & $(31,280)$ & $(31,889)$ & $1.9 \%$ & & $(28,871)$ & $(29,513)$ & $2.2 \%$ \\
\hline$\rho$ & & (3163) & (3277) & $3.6 \%$ & & (2644) & (2731) & $3.3 \%$ \\
\hline PV & & 0 & 0 & - & & 0 & 0 & - \\
\hline$\Delta$ & & 539,073 & 546,357 & $1.4 \%$ & & 539,073 & 546,357 & $1.4 \%$ \\
\hline$\Gamma$ & $0 \%$ & 11,539 & 11,681 & $1.2 \%$ & $0 \%$ & 11,539 & 11,681 & $1.2 \%$ \\
\hline$v$ & & 779,445 & 789,843 & $1.3 \%$ & & 779,445 & 789,843 & $1.3 \%$ \\
\hline$\Theta$ & & $(22,051)$ & $(22,461)$ & $1.9 \%$ & & $(22,051)$ & $(22,461)$ & $1.9 \%$ \\
\hline$\rho$ & & (1857) & (1917) & $3.2 \%$ & & (1857) & (1917) & $3.2 \%$ \\
\hline PV & & $(4,066,176)$ & $(4,130,919)$ & $1.6 \%$ & & $(3,441,668)$ & $(3,496,165)$ & $1.6 \%$ \\
\hline$\Delta$ & & 303,499 & 307,179 & $1.2 \%$ & & 385,400 & 390,802 & $1.4 \%$ \\
\hline$\Gamma$ & $(10 \%)$ & 6821 & 6903 & $1.2 \%$ & (6\%) & 10,854 & 11,009 & $1.4 \%$ \\
\hline$v$ & & 502,308 & 508,778 & $1.3 \%$ & & 646,507 & 655,964 & $1.5 \%$ \\
\hline$\Theta$ & & $(13,237)$ & $(13,437)$ & $1.5 \%$ & & $(14,707)$ & $(14,973)$ & $1.8 \%$ \\
\hline$\rho$ & & (873) & (881) & $0.9 \%$ & & (1018) & (1041) & $2.3 \%$ \\
\hline PV & & $(7,059,600)$ & $(7,161,419)$ & $1.4 \%$ & & $(7,010,772)$ & $(7,115,158)$ & $1.5 \%$ \\
\hline$\Delta$ & & 124,435 & 125,915 & $1.2 \%$ & & 145,788 & 147,574 & $1.2 \%$ \\
\hline$\Gamma$ & (25\%) & 2639 & 2674 & $1.3 \%$ & $(12 \%)$ & 5747 & 5827 & $1.4 \%$ \\
\hline$v$ & & 222,614 & 225,434 & $1.3 \%$ & & 339,955 & 344,633 & $1.4 \%$ \\
\hline$\Theta$ & & (5563) & (5639) & $1.4 \%$ & & (5420) & (5486) & $1.2 \%$ \\
\hline$\rho$ & & (291) & (285) & (2.1\%) & & (246) & (238) & (3.3\%) \\
\hline
\end{tabular}

$\dagger$ refers to the money-ness of the NYMEX WTI forward prices, not the delta of the Asian caps; †† refers to the money-ness of the NYMEX WTI implied volatilities, not the delta of the Asian caps. 
Table 9. Recent (as of September 19, 2013).

\begin{tabular}{|c|c|c|c|c|c|c|c|c|}
\hline \multirow[b]{2}{*}{ Attribute } & \multicolumn{4}{|c|}{ Forward Prices } & \multicolumn{4}{|c|}{ Implied Volatilities } \\
\hline & Money-ness $^{\dagger}$ & LIBOR & OIS & \% Variation & Money-ness ${ }^{\dagger \dagger}$ & LIBOR & OIS & \% Variation \\
\hline PV & & $23,804,754$ & $23,894,039$ & $0.4 \%$ & & $9,135,297$ & $9,189,343$ & $0.6 \%$ \\
\hline$\Delta$ & & $1,360,153$ & $1,365,324$ & $0.4 \%$ & & 811,904 & 817,437 & $0.7 \%$ \\
\hline$\Gamma$ & $25 \%$ & 12,825 & 12,893 & $0.5 \%$ & $12 \%$ & 14,451 & 14,532 & $0.6 \%$ \\
\hline$v$ & & 799,281 & 803,724 & $0.6 \%$ & & 787,310 & 793,132 & $0.7 \%$ \\
\hline$\Theta$ & & $(14,094)$ & $(14,228)$ & $1.0 \%$ & & $(32,071)$ & $(32,249)$ & $0.6 \%$ \\
\hline$\rho$ & & (3667) & (3686) & $0.5 \%$ & & (2552) & (2603) & $2.0 \%$ \\
\hline PV & & $7,201,572$ & $7,235,939$ & $0.5 \%$ & & $3,436,230$ & $3,456,985$ & $0.6 \%$ \\
\hline$\Delta$ & & 983,969 & 988,207 & $0.4 \%$ & & 682,866 & 686,417 & $0.5 \%$ \\
\hline$\Gamma$ & $10 \%$ & 20,687 & 20,777 & $0.4 \%$ & $6 \%$ & 19,048 & 19,129 & $0.4 \%$ \\
\hline$v$ & & 842,696 & 847,447 & $0.6 \%$ & & 737,968 & 742,399 & $0.6 \%$ \\
\hline$\Theta$ & & $(16,974)$ & $(17,082)$ & $0.6 \%$ & & $(22,753)$ & $(22,867)$ & $0.5 \%$ \\
\hline$\rho$ & & (1856) & (1888) & $1.7 \%$ & & (1426) & (1447) & $1.5 \%$ \\
\hline PV & & 0 & 0 & - & & 0 & 0 & - \\
\hline$\Delta$ & & 539,229 & 541,530 & $0.4 \%$ & & 539,229 & 541,530 & $0.4 \%$ \\
\hline$\Gamma$ & $0 \%$ & 22,715 & 22,796 & $0.4 \%$ & $0 \%$ & 22,715 & 22,796 & $0.4 \%$ \\
\hline$v$ & & 638,717 & 642,017 & $0.5 \%$ & & 638,717 & 642,017 & $0.5 \%$ \\
\hline$\Theta$ & & $(16,078)$ & $(16,141)$ & $0.4 \%$ & & $(16,078)$ & $(16,141)$ & $0.4 \%$ \\
\hline$\rho$ & & (724) & (732) & $1.1 \%$ & & (724) & (732) & $1.1 \%$ \\
\hline PV & & $(3,057,101)$ & $(3,072,304)$ & $0.5 \%$ & & $(2,693,153)$ & $(2,708,475)$ & $0.6 \%$ \\
\hline$\Delta$ & & 176,698 & 177,470 & $0.4 \%$ & & 327,910 & 328,914 & $0.3 \%$ \\
\hline$\Gamma$ & $(10 \%)$ & 8519 & 8553 & $0.4 \%$ & $(6 \%)$ & 23,948 & 24,013 & $0.3 \%$ \\
\hline$v$ & & 306,876 & 308,386 & $0.5 \%$ & & 423,468 & 425,320 & $0.4 \%$ \\
\hline$\Theta$ & & (5989) & (6012) & $0.4 \%$ & & (9072) & (9097) & $0.3 \%$ \\
\hline$\rho$ & & (230) & (229) & $-0.4 \%$ & & (218) & (217) & $(0.5 \%)$ \\
\hline PV & & $(4,251,746)$ & $(4,272,380)$ & $0.5 \%$ & & $(4,404,477)$ & $(4,426,127)$ & $0.5 \%$ \\
\hline$\Delta$ & & 35,489 & 35,716 & $0.6 \%$ & & 85,844 & 85,967 & $0.1 \%$ \\
\hline$\Gamma$ & (25\%) & 1592 & 1603 & $0.7 \%$ & $(12 \%)$ & 20,633 & 20,663 & $0.1 \%$ \\
\hline$v$ & & 73,962 & 74,428 & $0.6 \%$ & & 82,632 & 82,930 & $0.4 \%$ \\
\hline$\Theta$ & & (1158) & (1164) & $0.5 \%$ & & (1512) & (1514) & $0.1 \%$ \\
\hline$\rho$ & & (44) & (44) & $0.0 \%$ & & (9) & (9) & $0.0 \%$ \\
\hline
\end{tabular}

$\dagger$ refers to the money-ness of the NYMEX WTI forward prices, not the delta of the Asian caps; †† refers to the money-ness of the NYMEX WTI implied volatilities, not the delta of the Asian caps. 


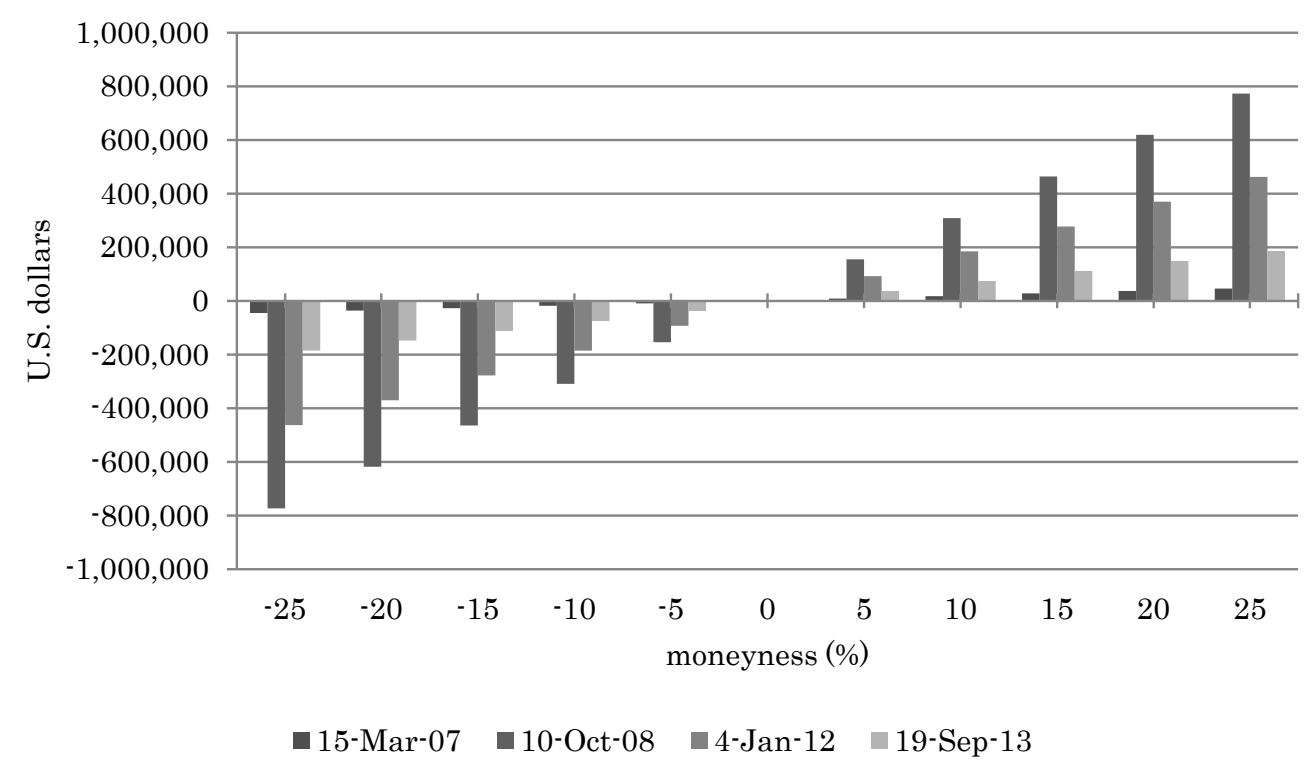

Figure 12. Differences in valuation between LIBOR discounting and OIS discounting.

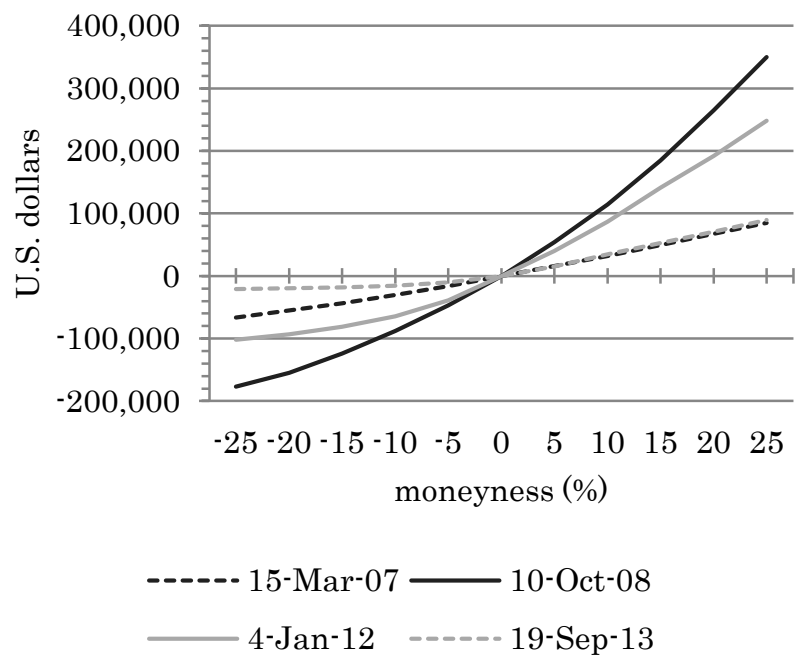

Figure 13. Differences in valuation, with forwards adjusted.

As Figure 14 depicts, when the implied volatilities of NYMEX WTI are shocked $\pm 15 \%$ in absolute terms, the impact on the valuation of the Asian cap becomes noticeable as implied volatility increases and decreases, and as the spread between LIBOR and OIS widens.

The impact to valuation of migrating from LIBOR to OIS discounting is likely to be limited for market participants with hedged portfolios that contain a large number of offsetting positions (e.g. banks). There is likely to be larger impact on directional portfolios containing transactions that hedge either consumption or production (e.g. end users).

We would like to emphasize that the figures presented in Tables 6-9 represent the impact to a single representative transaction. When considered in terms of a portfolio of transactions, the impact could be particularly significant given the level of the spread between LIBOR and OIS and the money-ness of the transactions in a directional portfolio.

To summarize, under stressed market conditions, the spread between LIBOR and OIS tends to widen. The impact on valuation can be particularly noticeable as the spread widens, especially for trades that are significantly in-the-money or out-of-the-money. Generally, the difference in valuation between the two discounting techniques will be more noticeable in transactions with long-dated tenors. 

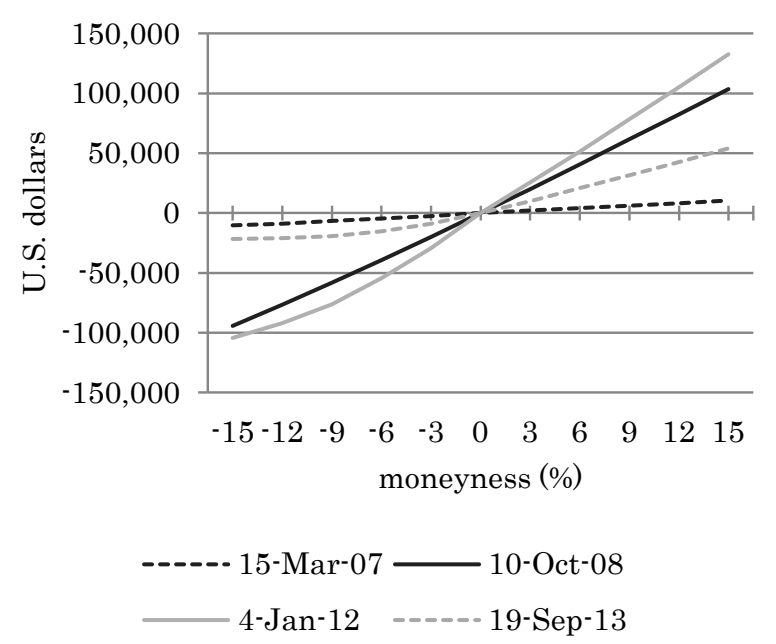

Figure 14. Differences in valuation, with volatilities adjusted.

\subsection{Impact on Risk Sensitivities}

The impact of switching from LIBOR to OIS discounting is not limited to valuation. The switch to OIS discounting can also materially impact the risk sensitivities of commodity swaps and options. Here, we examine the impact on the delta of a swap and an Asian cap, as well as the impact on the gamma, vega, theta and rho of an Asian cap.

Figure 15 depicts the change in the swaps' delta in the pre-crisis, peak-crisis, post-crisis and recent market environments given varying levels of money-ness in the forward prices of crude oil. The results here are intuitive. Due to the linearity of this instrument, the change in delta is constant across varying levels of money-ness in the forward prices of crude oil.

Figure 16 depicts the change in the Asian caps' delta given varying levels of money-ness in the forward prices of crude oil. In this scenario, the change in delta as a result of switching from LIBOR to OIS discounting becomes noticeable as the option moves progressively in-the-money (i.e. as the delta approaches one) and as the spread between LIBOR and OIS widens.

Figure 17 depicts the change in the Asian caps' delta given varying levels of money-ness in the implied volatilities of crude oil. In this scenario, the change in delta as a result of switching from LIBOR to OIS discounting becomes noticeable as the absolute level of implied volatility increases (which, in turn, causes the option's delta to approach one) and as the spread between LIBOR and OIS widens.

Figure 18 depicts the change in the Asian caps' gamma given varying levels of money-ness in the forward prices of crude oil. In this scenario, the change in gamma as a result of switching from LIBOR and OIS discounting becomes noticeable as the option moves progressively out-the-money and as the spread between LIBOR and OIS widens.

Figure 19 depicts the change in the Asian caps' gamma given varying levels of money-ness in the implied volatilities of crude oil. In this scenario, the change in gamma as a result of switching from LIBOR to OIS discounting becomes noticeable as the spread between LIBOR and OIS widens, but is not generally influenced by the absolute level of implied volatility.

Figure 20 depicts the change in the Asian caps' vega given varying levels of money-ness in the forward prices of crude oil. In this scenario, the change in vega as a result of switching from LIBOR to OIS discounting becomes noticeable as the option moves progressively in-the-money (i.e. as the delta approaches one) and as the spread between LIBOR and OIS widens.

Figure 21 depicts the change in the Asian caps' vega given varying levels of money-ness in the implied volatilities of NYMEX WTI. In this scenario, the change in vega as a result of switching from LIBOR to OIS discounting becomes noticeable as the absolute level of implied volatility increases, thereby causing the option to move progressively in-the-money (i.e. as the delta approaches one) and as the spread between LIBOR and OIS widens.

Figure 22 depicts the change in the options' theta given varying levels of money-ness in the forward prices of 


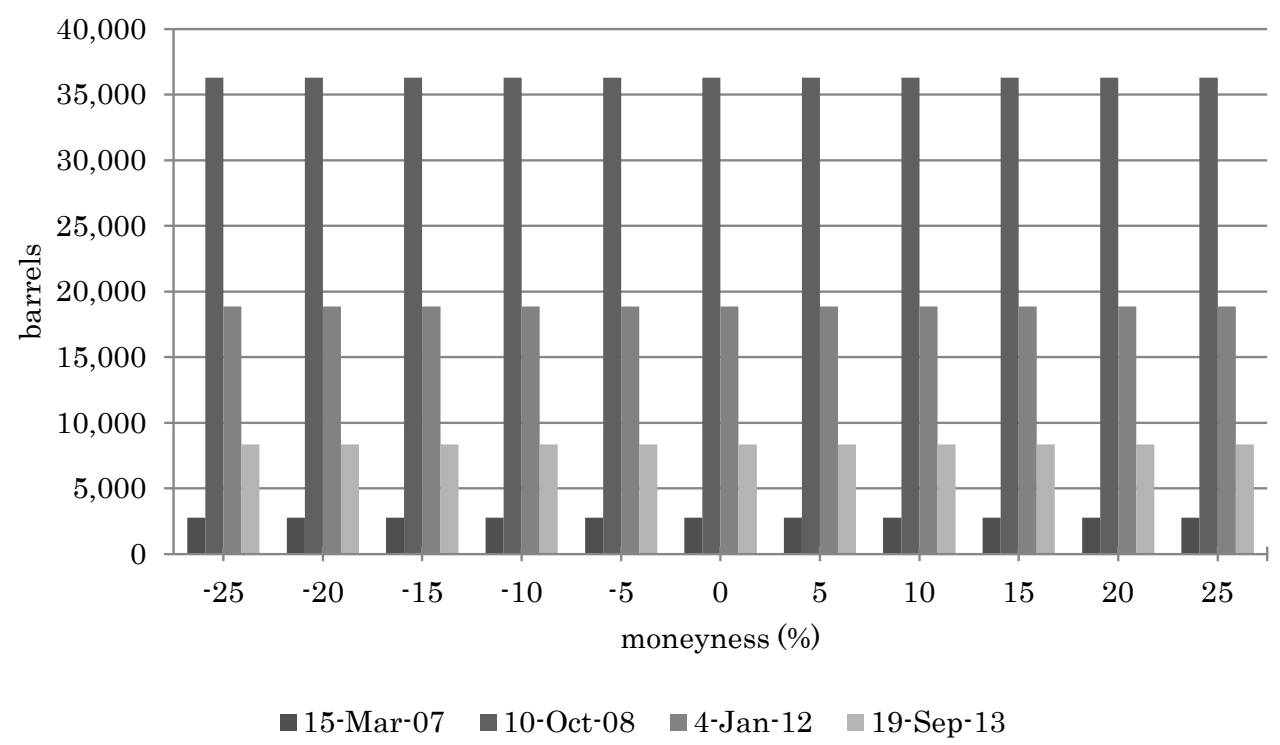

Figure 15. Differences in delta between LIBOR discounting and OIS discounting.
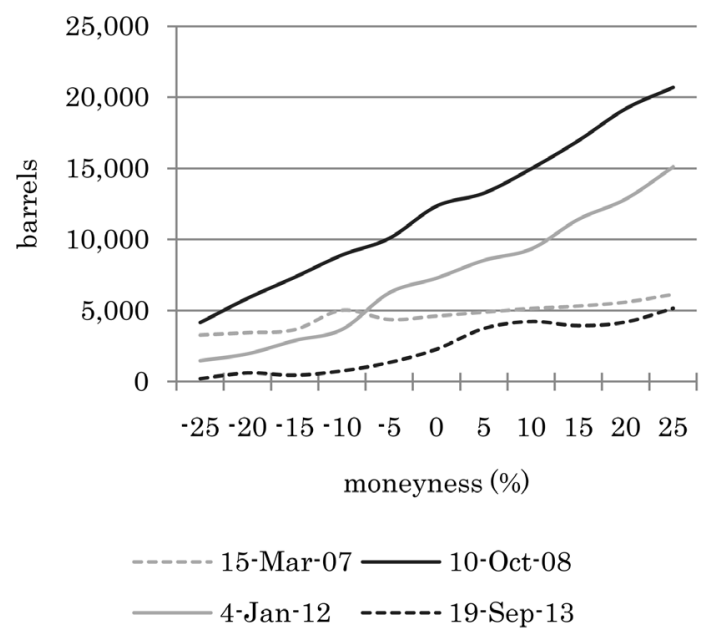

Figure 16. Differences in delta, with forward prices adjusted.

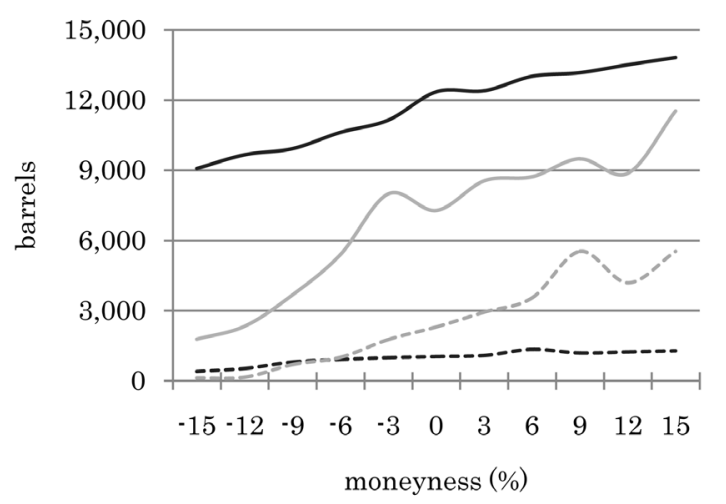

\begin{tabular}{l}
------ 15-Mar-07 - 10-Oct-08 \\
\hline 4-Jan-12 ------ 19-Sep-13
\end{tabular}

Figure 17. Differences in delta, with volatilities adjusted. 


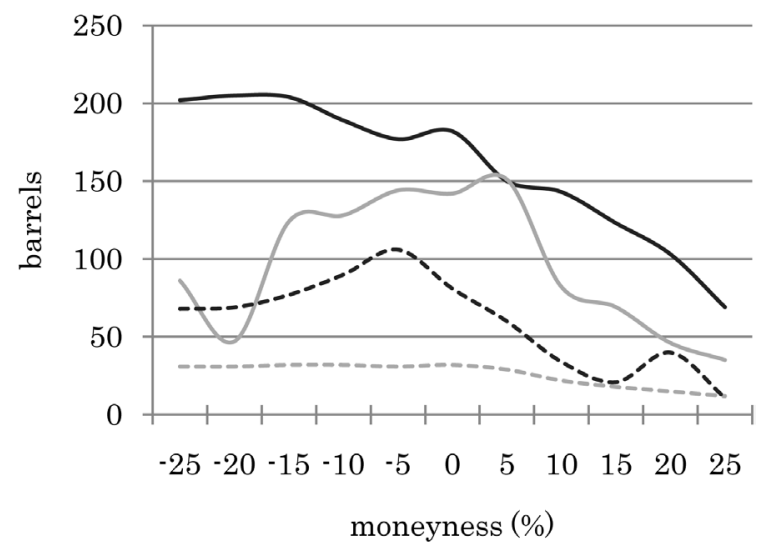

$$
\begin{array}{r}
\text { 15-Mar-07 } 10 \text {-Oct-08 } \\
\text { 4-Jan-12 ------ 19-Sep-13 }
\end{array}
$$

Figure 18. Differences in gamma, with forwards adjusted.

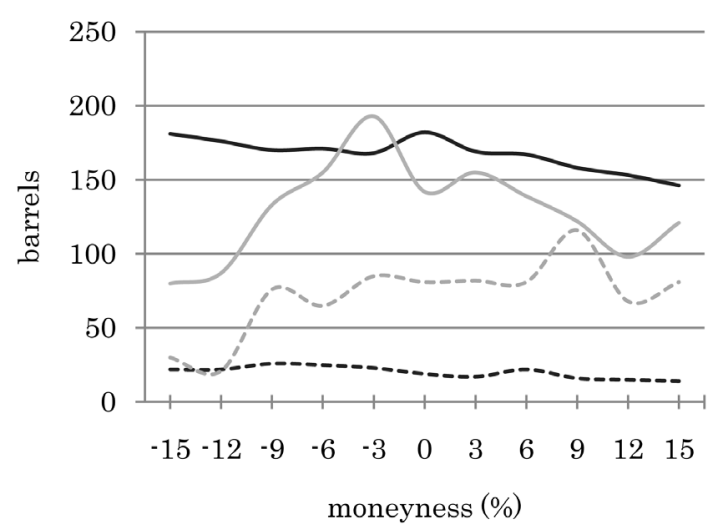

$$
\begin{aligned}
& \text {----- 15-Mar-07 10-Oct-08 } \\
& \text { 4-Jan-12 ------- 19-Sep-13 }
\end{aligned}
$$

Figure 19. Differences in gamma, with volatilities adjusted.

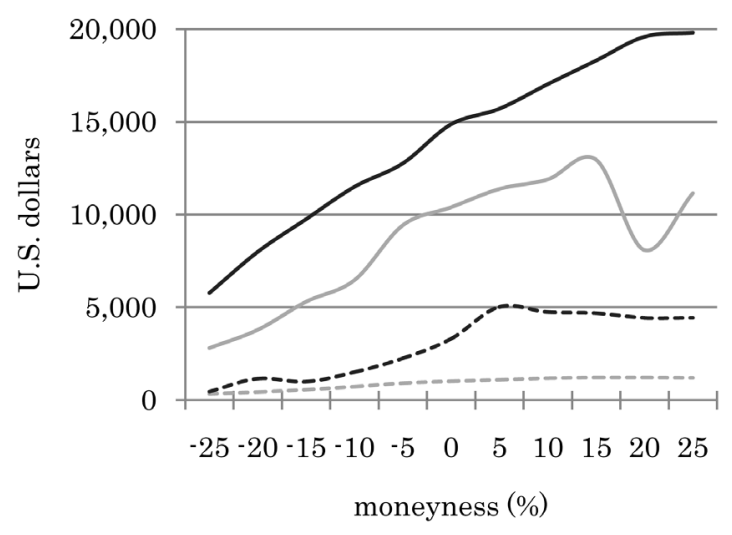

$$
\begin{aligned}
& \text {------ 15-Mar-07 10-Oct-08 } \\
& \text { 4-Jan-12 ------ 19-Sep-13 }
\end{aligned}
$$

Figure 20. Differences in vega, with forwards adjusted. 


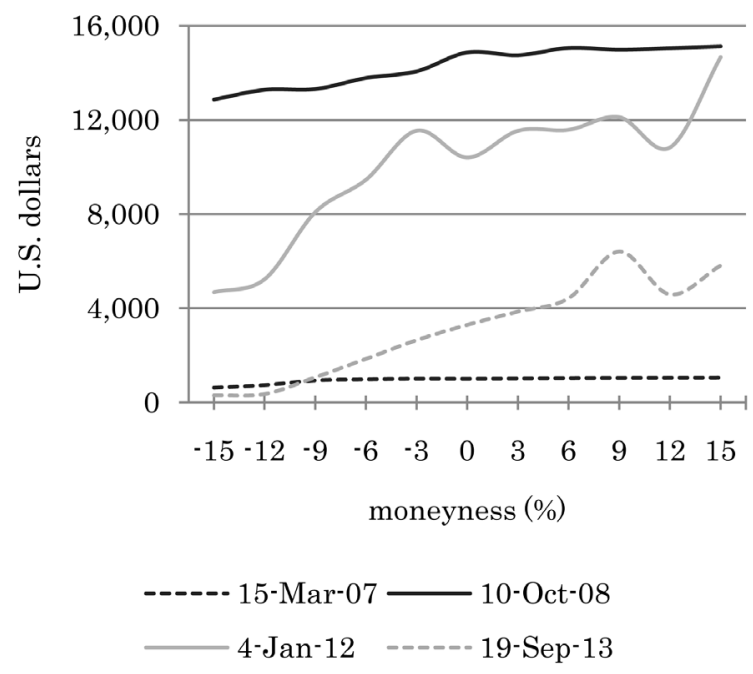

Figure 21. Differences in vega, with volatilities adjusted.

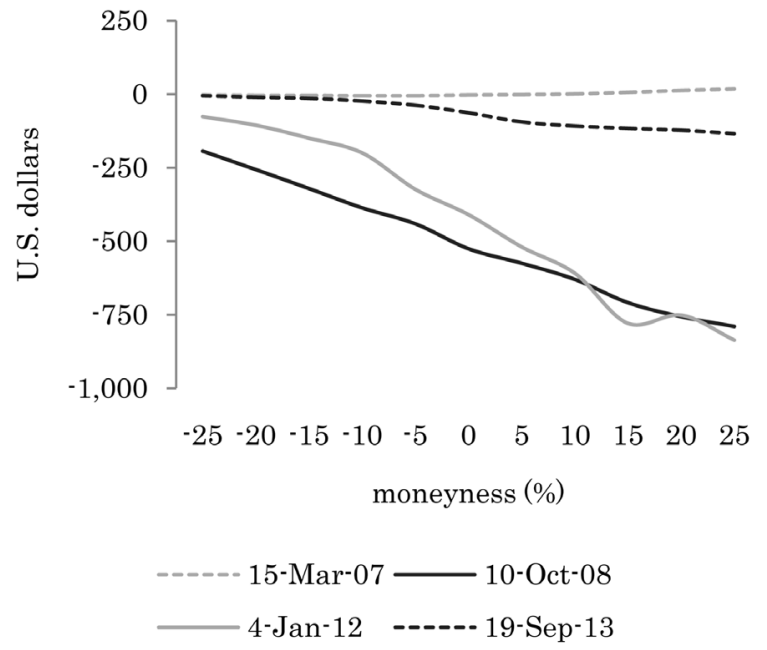

Figure 22. Differences in theta, with forwards adjusted.

crude oil. In this scenario, the change in theta as a result of switching from LIBOR to OIS discounting becomes noticeable as the option moves progressively in-the-money and as the spread between LIBOR and OIS widens.

Figure 23 depicts the change in the options' vega given varying levels of money-ness in the implied volatilities of crude oil. In this scenario, the change in vega as a result of switching from LIBOR to OIS discounting becomes noticeable as the absolute level of implied volatility increases, thereby causing the option to move progressively in-the-money (i.e. as the delta approaches one) and as the spread between LIBOR and OIS widens.

Figure 24 depicts the change in the options' rho given varying levels of money-ness in the forward prices of crude oil. In this scenario, the change in rho as a result of switching from LIBOR to OIS discounting becomes noticeable as the option moves progressively in-the-money and as the spread between LIBOR and OIS widens.

Figure 25 depicts the change in the options' rho given varying levels of money-ness in the implied volatilities of crude oil. In this scenario, the change in rho as a result of switching from LIBOR to OIS discounting becomes noticeable as the absolute level of implied volatility increases, thereby causing the option to move progressively in-the-money (i.e. as the delta approaches one) and as the spread between LIBOR and OIS widens.

\section{Impact on Hedge Accounting}

On June 26, 2013, the Emerging Issues Task Force (EITF) of the Financial Accounting Standards Board (FASB) ratified its earlier consensus view that the effective Federal Funds rate (and, by extension, the OIS rates) would 


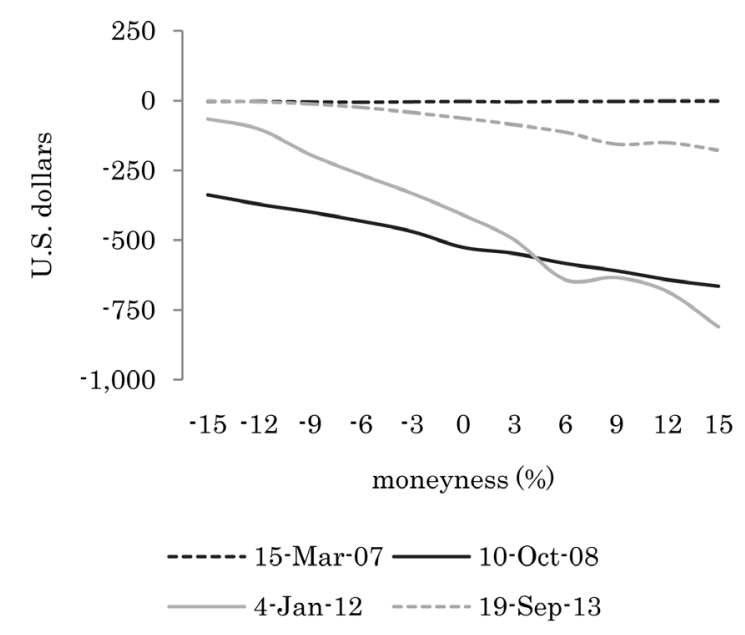

Figure 23. Differences in theta, with volatilities adjusted.

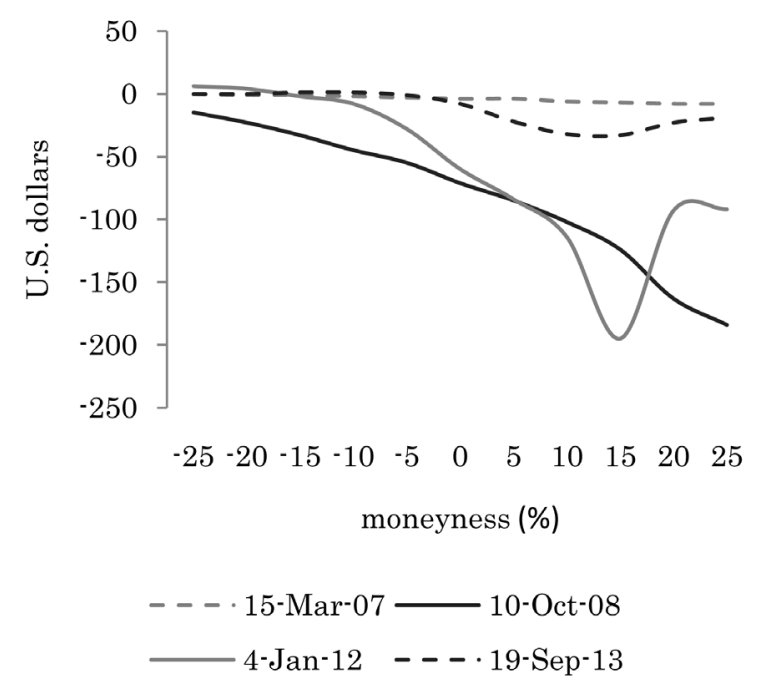

Figure 24. Differences in rho, with forwards adjusted.

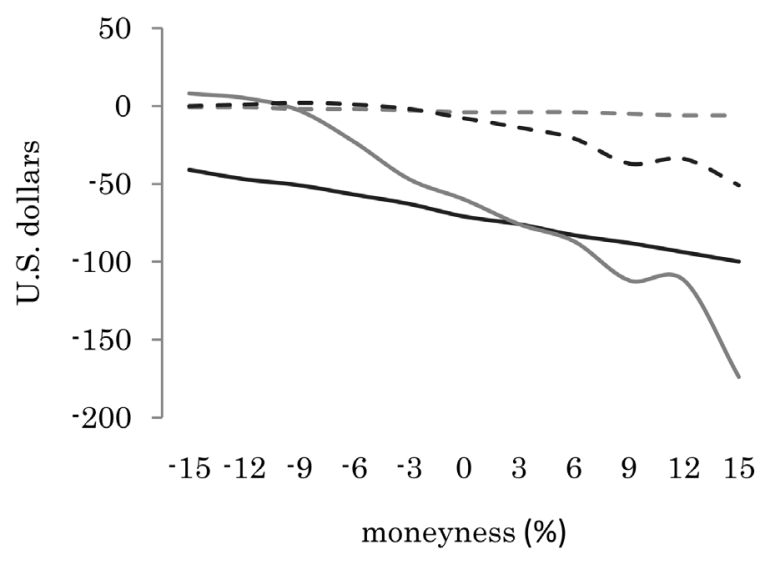

$$
\begin{aligned}
& ---\cdot \text { 15-Mar-07 10-Oct-08 } \\
& \text { 4-Jan-12 - - - 19-Sep-13 }
\end{aligned}
$$

Figure 25. Differences in rho, with volatilities adjusted. 
be included as a benchmark interest rate for hedge accounting purposes in the United States.

Accounting Standards Codification (ASC) Topic 820 (formerly Statement of Financial Accounting Standards 157) defines fair value as "the price that would be received to sell an asset or paid to transfer a liability in an orderly transaction between market participants at the measurement date." Using the OIS curve as an input more accurately reflects fair value of collateralized derivative contracts.

Under Generally Accepted Accounting Principles (GAAP) in the U.S., the change in valuation methodology is treated prospectively as a change in estimate. According to ASC 820-10-35-25 (FASB ASC 820-10-35-25), "... a change in a valuation technique... is appropriate if the change results in a measurement that is equally or more representative of fair value in the circumstances.” ASC 820-10-35-25 (FASB ASC 820-10-35-25) cites several examples, including recent developments in the marketplace and improved valuation techniques, both of which apply in the case where an entity switches from LIBOR discounting to OIS discounting.

One key question is whether it is appropriate to use the OIS discount factors to value the cash flows of a hypothetical derivative in a cash flow hedge relationship in accordance with ASC 815. In a cash flow hedge, the same discount factors (either OIS or LIBOR) are applied to both the derivative hedge and the hedged item. Ineffectiveness in cash flow hedges is not likely to be impacted.

Another key question is whether the switch to OIS will require de-designation of the original hedge relationship. De-designation is not required if the change does not conflict with the hedge designation documentation and the critical aspects of the hedge relationship remain intact.

\section{Organizational Impacts and the Reluctance to Switch}

Switching from LIBOR discounting to OIS discounting would affect various functions within an organization, including risk management, treasury and technology. The risk management function would need to quantify and monitor the effects of additional risk factors, including OIS rates and the spread between LIBOR and OIS (depending on the nature of the collateral agreements). The treasury function would need to monitor and optimize the firm's use of collateral. Systems would need to be modified to take into account new discount curves, distinguish between collateralized trades and non-collateralized trades and link trades to collateral details.

Many corporations are reluctant to switch from LIBOR to OIS discounting when portfolio valuations experience a sudden, and potentially significant, gain or loss, which would likely prompt questions. It might be difficult to explain the discontinuity. Therefore many corporations favor the pre-crisis, status quo methodology. Even if they understand the appropriateness of using OIS rates for pricing and risk management, switching to a new discounting methodology might require significant changes to technology and/or back office infrastructure, both of which could come at a material cost.

\section{Conclusion}

Prior to the financial crisis, the spread between LIBOR and OIS was negligible. LIBOR was used as a proxy for the risk-neutral discount rate, even if some market participants realized the appropriateness of using OIS rates instead. During the financial crisis, the spread between LIBOR and OIS widened significantly, calling into question the appropriateness of using LIBOR to price default-free cash flows in derivatives contracts. Market participants were reminded that LIBOR and OIS rates shared different credit and liquidity characteristics, with many adopting the use of OIS rates for pricing and risk management of commodity derivatives.

Since the financial crisis, the spread between LIBOR and OIS has narrowed to more traditional levels, but remains elevated relative to the pre-crisis environment due to concerns over credit risk and liquidity. Practitioners in the interest rate markets have largely abandoned LIBOR and have embraced the use of OIS discounting. Practitioners in the commodities markets have been slower to adopt this post-crisis technique. According to the June 2013 ISDA Margin Survey (International Swaps and Derivatives Association, Inc., June 2013), approximately $70 \%$ of participants in the commodities markets continue to use LIBOR for pricing and risk management.

To our knowledge, the most leading market-making banks have adopted the use of OIS discounting. Until there is more consistency among asset classes, many non-financial corporations will continue to use the LIBOR curve for valuation purposes. However, if the spread between LIBOR and OIS were to widen, these firms would be required by the Dodd-Frank Act to reconcile valuation differences and to report these differences to regulators. Moreover, from an accounting perspective, the impact to a corporation's cash flow hedge accounting is likely to be limited since hypothetical derivatives are applied. 
In the case of linear instruments (e.g. swaps), the difference in valuation between LIBOR and OIS is magnified as the spread between LIBOR and OIS widens and as the trade's valuation moves further in-the-money or out-of-the-money. In the case of non-linear instruments (e.g. Asian caps), the difference in valuation mirrors that of linear instruments, but is magnified as a trade's delta approaches one. We observe similar effects on an Asian cap's Greek sensitivities, with the exception of gamma.

There is no question as to the appropriateness of using the OIS curve to value collateralized derivatives transactions. However, the debate continues as to whether it is appropriate to include a funding value adjustment in derivatives pricing, a view that conflicts with that of Hull and White.

It is our view that the Law of One Price no longer applies. Banks that are able to fund at favorable levels can offer their clients more attractive trade prices than competing banks funding at comparatively higher levels, ceteris paribus. The appropriate discount curve depends on the creditworthiness of one's self and one's counterparty, along with the netting and collateral clauses in the CSA agreements.

Given the variability that could result between LIBOR and OIS discounting, commodity market participants are urged to clarify the valuation approaches used by their counterparties. In particular, end users should understand precisely their CSA provisions and associated valuation methodology that will be applied to a transaction not only at the time of execution, but also at the time of amendment, assignment or unwinding. The consequences of not doing so could result in profitable arbitrage opportunities for one's counterparty.

\section{Acknowledgements}

The author gratefully acknowledges the suggestions provided by Dr. William O. Smith.

\section{References}

Bank for International Settlements (2011). Liquidity Transfer Pricing: A Guide to Better Practice. Retrieved January 14, 2014, from Bank for International Settlements. http://www.bis.org/fsi/fsipapers10.pdf

Bloomberg, L. P. (2014). 3-Month U.S. Dollar Overnight Index Swap. 04-Dec-2001 to 27-Jan-2014. Retrieved from Bloomberg Database.

Brigo, D., \& Masetti, M. (2005). Risk-Neutral Pricing of Counterparty Risk. In M. Pykhtin (Ed.), Counterparty Credit Risk Modeling: Risk Management, Pricing and Regulation. London: Risk Books.

Cameron, M. (2013). Goldman and the OIS Gold Rush: How Fortunes Were Made from a Discounting Change. http://www.risk.net/risk-magazine/feature/2270178/goldman-and-the-ois-gold-rush-how-fortunes-were-made-from-a-disc ounting-change

Canabarro, E., Picoult, E., \& Wilde, T. (2005). Counterparty Risk, Energy Risk, May Issue.

FASB ASC 820-10-35-25

Gabillion, J. (1991). The Term Structure of Oil Futures Prices. Working Paper, No. M17, Oxford Institute for Energy Studies.

Hull, J., \& White, A. (2012). LIBOR vs. OIS: The Derivatives Discounting Dilemma. Journal of Investment Management, $11,14-27$.

International Swaps and Derivatives Association, Inc. (2013, June 21). ISDA Margin Survey 2013. http://www2.isda.org/functional-areas/research/surveys/margin-surveys/

International Swaps and Derivatives Association, Inc. (2013, October 23). 2013 Best Practices for OTC Derivatives Collateral Process. http://www2.isda.org/functional-areas/infrastructure-management/collateral/

Mollencamp, C. (2008). Bankers Cast Doubt on Key Rate Amid Crisis. The Wall Street Journal: http://online.wsj.com/

Pallavicini, A., Perini, D., \& Brigo, D. (2011). Funding Valuation Adjustment: A Consistent Framework Including CVA, DVA, Collateral, Netting Rules and Re-Hypothecation. http://ssrn.com

Securities and Exchange Commission (2011). SEC Response Letter. http://www.sec.gov/Archives/edgar/data/895421/000119312511162061/filename1.htm

Sorenson, E. H., \& Bollier, T. F. (1994). Pricing Swap Default Risk. Financial Analysts Journal, 50, $23-33$. http://dx.doi.org/10.2469/faj.v50.n3.23

Turnbull, S., \& Wakeman, L. (1991). A Quick Algorithm for Pricing European Average Options. Journal of Financial and Quantitative Analysis, 26, 377-389. http://dx.doi.org/10.2307/2331213 


\section{Appendix A}

A rigorous derivation of the pricing equation for a financial derivative contract requires application of the Feynman-Kac theorem ${ }^{8}$. We derive the pricing equation with a simpler approach, which is perfectly adequate.

Firstly, we assume that the financial derivative contract has a single Markovian underlying process that follows

$$
\mathrm{d} S=a(S, t) \mathrm{d} t+b(S, t) \mathrm{d} W_{t}
$$

We further assume that this process is in the risk-neutral measure $\mathbb{Q}$.

By Ito's lemma, the stochastic differential equation for the price of a derivative contract with an underlying process $S$ is

$$
\mathrm{d} G=\frac{\partial G}{\partial t} \mathrm{~d} t+a(S, t) \frac{\partial G}{\partial \mathrm{s}} \mathrm{d} t+\frac{1}{2} b(s, t)^{2}+\frac{\partial^{2} G}{\partial s^{2}} \mathrm{~d} t+b(s, t) \frac{\partial G}{\partial s} \mathrm{~d} W
$$

Given the assumption that we are pricing in the risk-neutral world, the expected relative rate of return over an infinitesimal period of time is the risk-free rate.

Now, we take the expectation of $\mathrm{d} G$

$$
E_{0}[\mathrm{~d} G]=E_{0}\left[\frac{\partial G}{\partial t}+a(S, t) \frac{\partial G}{\partial s}+\frac{1}{2} b(s, t)^{2}+\frac{\partial^{2} G}{\partial s^{2}}\right] \mathrm{d} t+E_{0}\left[b(s, t) \frac{\partial G}{\partial s} \mathrm{~d} W\right]
$$

Which gives us

$$
E_{0}[\mathrm{~d} G]=r G \mathrm{~d} t
$$

Since

$$
E_{0}\left[b(s, t) \frac{\partial G}{\partial s} \mathrm{~d} W\right]=b(s, t) \frac{\partial G}{\partial s} E_{0}[\mathrm{~d} W]=0
$$

We have

$$
\frac{\partial G}{\partial t}+a(S, t) \frac{\partial G}{\partial s}+\frac{1}{2} b(s, t)^{2}+\frac{\partial^{2} G}{\partial s^{2}}=r G
$$

In Equation (6), we have a partial differential equation for the price of the contract, where we interpret as the possible realizations of $S$.

This equation has suitable end conditions that reflect the value of the contract at maturity.

If the contract matures at time $T$ with a payoff $F(S ; K)$, where $K$ is a parameter such as strike, then the end condition is

$$
G(s, t=T)=F(s ; K)
$$

${ }^{8}$ The most famous application of this theorem is for deriving the Black-Scholes formula, when $X_{t}$ is the underlying stock price, $\mu(x, t)=r(x, t)=g\left(X_{t}\right)=\operatorname{MAX}\left(S_{t}-K, 0\right)$. 
Scientific Research Publishing (SCIRP) is one of the largest Open Access journal publishers. It is currently publishing more than 200 open access, online, peer-reviewed journals covering a wide range of academic disciplines. SCIRP serves the worldwide academic communities and contributes to the progress and application of science with its publication.

Other selected journals from SCIRP are listed as below. Submit your manuscript to us via either submit@scirp.org or Online Submission Portal.
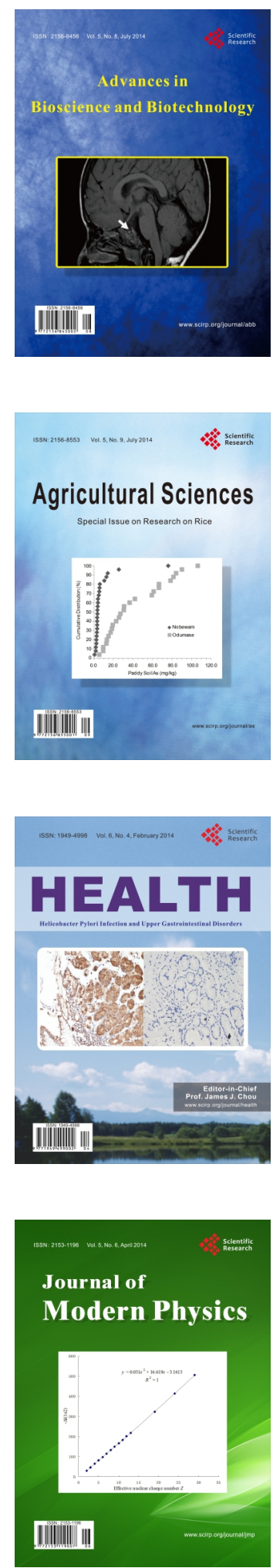
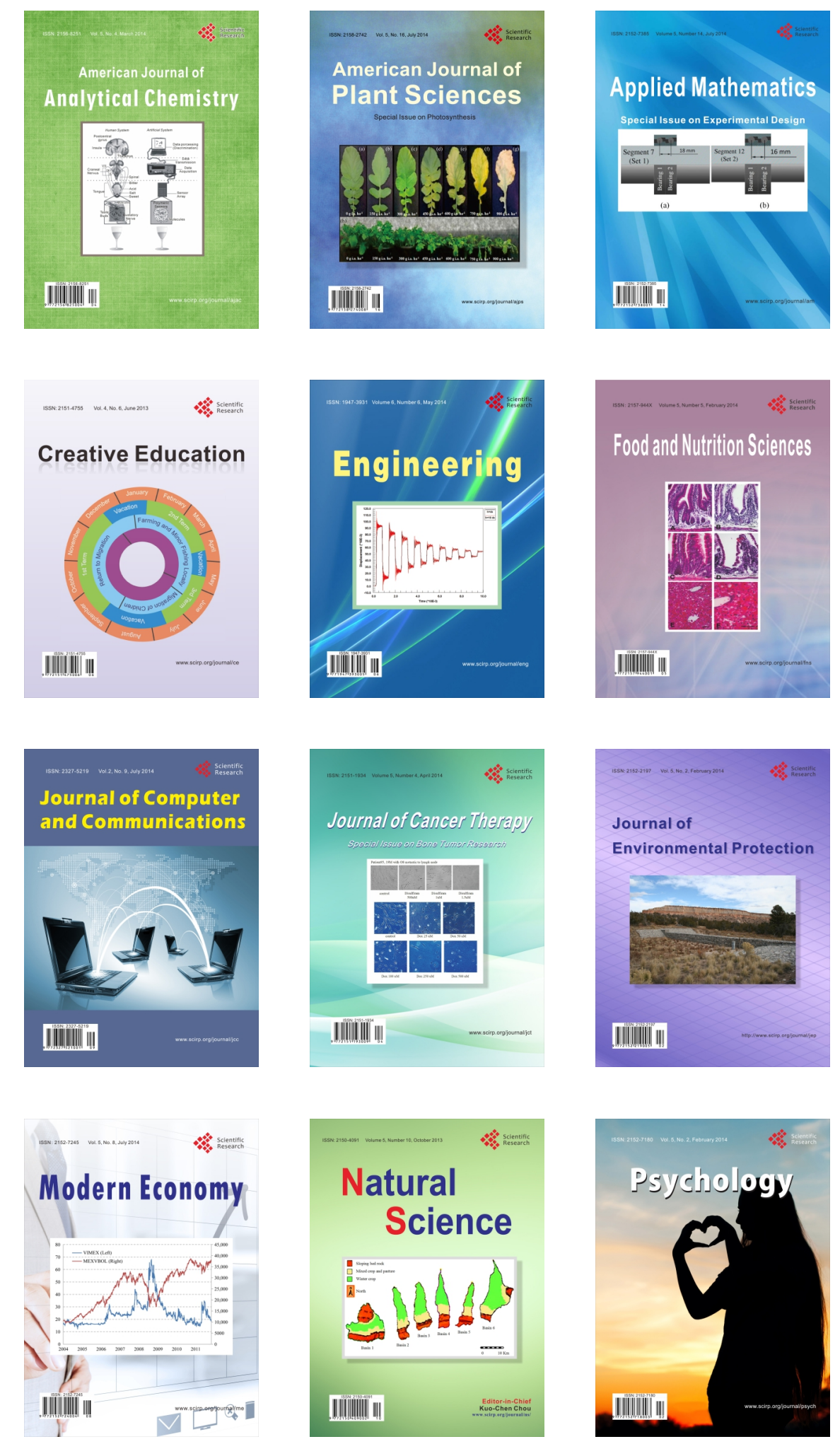